\title{
Attention control processes that prioritise task execution may come at the expense of incidental memory encoding
}

\author{
Lewis Dunne*, Bertram Opitz \\ University of Surrey, GU2 7XH, United Kingdom
}

\section{A R T I C L E I N F O}

\section{Keywords:}

Attention

Top-down

Bottom-up

Encoding

Subsequent memory

\begin{abstract}
A B S T R A C T
Attention underpins episodic memory encoding by gating information processing. However, it is unclear how different forms of attention affect encoding. Using fMRI, we implemented a novel task that separates top-down and bottom-up attention (TDA; BUA) to test how these forms of attention influence encoding. Twenty-seven subjects carried out a scanned incidental encoding task that required semantic categorisation of stimuli. Trials either required visual search (TDA) to locate a target, or the target blinked and captured attention (BUA). After a retention period, subjects performed a surprise recognition test. Univariate analyses showed that ventral visual regions and right hippocampus indexed encoding success. Psychophysiological interaction analyses showed that, during TDA, there was increased coupling between dorsal parietal cortex and fusiform gyrus with encoding failure, and between lateral occipital cortex and fusiform gyrus with encoding success. No significant connectivity modulations were observed during BUA. We propose that increased TDA to objects in space is mediated by parietal cortex and negatively impacts encoding. Also, increases in connectivity within ventral visual cortex index the integration of stimulus features, promoting encoding. Finally, the influences of attention on encoding likely depend on task demands: as cognitive control increases, task execution is emphasised at the expense of memory encoding.
\end{abstract}

\section{Introduction}

Attention is crucial for successful memory encoding (Aly \& TurkBrowne, 2016; Naveh-Benjamin, Guez, Hara, Brubaker, \& LowenschussErlich, 2014; Uncapher \& Rugg, 2005), but memory also shapes how attention is allocated (Aly \& Turk-Browne, 2017; Bridge, Cohen, \& Voss, 2017; Humphrey \& Underwood, 2009; Summerfield, Lepsien, Gitelman, Mesulam, \& Nobre, 2006; West Chanon \& Hopfinger, 2008). Although this interplay is well documented, there has been comparatively less work addressing the questions that arise naturally from their mutual consideration. One such question concerns how different forms of attention might influence encoding and subsequent episodic retrieval. Understanding this interplay is a crucial step in theory development and is the focus of the present study.

The attention system is limited (Broadbent, 1958; Desimone \& Duncan, 1995; Schneider \& Shiffrin, 1977), and information competes for attention resources (Kastner \& Ungerleider, 2001; Ungerleider, 2000), with top-down attention (TDA) and bottom-up attention (BUA) mechanisms interacting to support orienting (Schreij, Theeuwes, \& Olivers, 2010; Theeuwes, 1991, 1992; Treisman \& Gelade, 1980; Treisman \& Gormican, 1988). With respect to their neural correlates, a functional-neuroanatomical distinction exists between the two modes of attention whereby TDA is served by a dorsal attention network (DAN), while BUA is served by a ventral attention network (VAN) (Corbetta, Patel, \& Shulman, 2008; Corbetta \& Shulman, 2002). The organisation of these networks is observable both during task-based (Bressler, Tang, Sylvester, Shulman, \& Corbetta, 2008; Corbetta, Kincade, Ollinger, McAvoy, \& Shulman, 2000; Corbetta, Kincade, \& Shulman, 2002; Hopfinger, Buonocore, \& Mangun, 2000) and restingstate fMRI paradigms (Bressler \& Menon, 2010; Fox, Corbetta, Snyder, Vincent, \& Raichle, 2006), and they form key components in networkbased models of brain function (Yeo et al., 2011).

Highly salient stimuli capture attention (Awh \& Jonides, 2001; Chun \& Turk-Browne, 2007; Corbetta et al., 2002; Gaspelin \& Luck, 2018; Gazzaley \& Nobre, 2012; Kerzel \& Schönhammer, 2013; Naghavi \& Nyberg, 2005; Santangelo \& Macaluso, 2013a), and lead to improved short-term/working memory (Fine \& Minnery, 2009; Santangelo \& Macaluso, 2013b) to the detriment of non-salient concurrently presented stimuli (Melcher \& Piazza, 2011), suggesting a myopic preference on the basis of salience. Further to this, salience is also known to benefit long-term memory, as demonstrated by the isolation effect which shows that salient stimuli embedded among homogenous (low-

\footnotetext{
* Corresponding author.

E-mail address: 1.dunne@surrey.ac.uk (L. Dunne).
} 
salience) stimuli are more memorable (Bireta \& Mazzei, 2016; Halamish, 2018; Schmidt \& Schmidt, 2017). Although still not fully understood, the isolation effect is likely due to increased attentional resources directed to distinctive stimuli, resulting in an encoding advantage. During a given task, shared properties of stimuli are spontaneously identified and contextualised within an existing semantic structure, allowing for category-level processing. However, when this context is violated by an isolate, increased attentional resources are required in order to process finer stimulus details. Notably, an fMRI experiment looking at the isolation effect showed that not only did salient isolates evoke activation of the cingulo-opercular "salience" network and the VAN, but they were also better remembered in a subsequent recall task (Strange, Henson, Friston, \& Dolan, 2000). Therefore, BUA processing of salient stimuli seems beneficial to encoding, but there is very little neuroimaging research into these effects.

A key dependent variable in the study of encoding is the subsequent memory effect (SME), representing differences in neural activity at encoding that predict later memory success and failure (for a review, see Paller \& Wagner, 2002). These can be increases in regional BOLD activity in response to items later remembered (remembered > forgotten; positive SMEs) or increases in response to items later forgotten (forgotten $>$ remembered; negative SMEs). In a meta-analysis of the overlap between attention and SMEs, it was found that positive SMEs occurred mainly in dorsal posterior parietal cortex (PPC), but that negative SMEs occurred mostly in ventral PPC, highlighting an overlap in the regions supporting attention and episodic encoding (Uncapher \& Wagner, 2009). By extension, given the dorsal-TDA and ventral-BUA distinction in the attention literature, the authors hypothesised that TDA may be beneficial whereas BUA may be detrimental to encoding. Using fMRI, the authors then tested this with a spatial cueing task adapted with pictorial stimuli (Uncapher, Hutchinson, \& Wagner, 2011) and, supporting their previous meta-analysis, TDA effects and positive SMEs overlapped in the DAN, whereas BUA effects and negative SMEs overlapped in the VAN. Also, TDA processing led to better recognition overall, and psychophysiological interaction (PPI) analyses showed that encoding was influenced by parietal interactions with visual object representation regions, with a positive influence from the DAN but a negative influence from the VAN. Importantly, these data are contrary to the predictions made by the previous literature on the effects of attention on memory, namely that information that captures attention in a bottom-up manner should receive preferential encoding.

The effects of TDA and BUA manipulations on episodic recognition memory are still yet to be extensively documented, and the contrasting findings from the isolation and cueing literature, with regards to encoding, are difficult to explain. The isolation effect presents a strong argument that attention capturing stimuli are better remembered because the use of perceptually salient isolates is a reasonable manipulation of BUA, yet it can say nothing about the influence of TDA. For this reason, it cannot be easily interpreted under the dual attention framework as this would require that both modes of attention are manipulated; the involvement of BUA mechanisms in response to isolates is not necessarily enough to achieve this. On the other hand, while the central cueing task was initially designed to demonstrate response time benefits due to expectation (presumed TDA) and response time costs when this expectancy was violated on invalid trials, it is generally acknowledged that these violations elicit BUA. However, it is unclear whether expectancy violation is the same as or distinct from presenting a stimulus with high perceptual salience. Similarly, the cueing task produces conflict when a target appears in the uncued location, but it is unclear whether conflict is intrinsic in BUA or if it is in fact a confound.

Here, we present a novel visual search task that separates TDA and BUA using meaningful pictorial stimuli as targets and distractors. Visual search represents an everyday example whereby TDA and BUA are engaged in order to identify a target stimulus (Treisman \& Gelade, 1980; Treisman \& Gormican, 1988). In order to test the effects of attention on memory, there should be a direct manipulation of attention mode between TDA and BUA. Moreover, stimulus-specific conflict also occurs in the presence of distracting stimuli. Searching an array of meaningful pictorial stimuli for a semantically-defined target object is an adaptation of the simple visual search paradigm, and is likely to produce responses in ventral visual regions of the brain that are sensitive to object processing (Bracci, Ritchie, \& de Beeck, 2017; Goddard, 2017; Grill-Spector \& Weiner, 2014). Furthermore, to standardise the salience of targets that "pop out" and capture attention, there is evidence suggesting that an abrupt stimulus onset or change (Forster \& Lavie, 2011; Schreij et al., 2010; Sunny \& von Mühlenen, 2013; Yantis \& Hillstrom, 1994; Yantis, 1993; Yantis \& Jonides, 1984) is an effective way in which attention can be captured via BUA mechanisms. To maximise this effect, the frequency of pop out stimuli can be reduced to increase their salience further, as low-frequency stimuli violate expectation (Han \& Marois, 2014; Kim, 2014; Posner, 2014; Sokolov, 1963). This manipulation between a standard search on the one hand, and an unexpected capture on the other, allows for a novel and direct manipulation of TDA and BUA that would help to shed light on how these attention mechanisms contribute to encoding. Although visual search may be explained by both top-down and bottom-up factors (Wolfe \& Horowitz, 2017), the searching of a visual array for a target is broadly a top-down process as it is governed by internal goals and requires cognitive control; the interruption of such a process by a stimulus that attracts attention is broadly bottom-up since the response is reflexive. Thus, in the current experiment, we operationalise TDA as sustained visual search and BUA as an interruption to ongoing processes by an attention capturing event. Given the importance of the visual search paradigm in our current understanding of attention, this would be a fitting addition to the small body of literature exploring the links between attention and memory.

Using fMRI to test how these forms of attention impact episodic encoding and later recognition, we hypothesised that search and capture trials will elicit responses in the DAN and VAN, respectively, and that neural activity at encoding that predicts subsequent memory (SM) performance would differ between TDA and BUA. In line with the isolation literature, which demonstrates a clearer BUA manipulation, we hypothesise that targets encoded via BUA will lead to better subsequent recognition performance than those encoded via TDA. To test this further, psychophysiological interaction (PPI) analyses will be carried out. Two analyses will use seeds in the left and right dorsal PPC (for TDA), respectively, while a third will use a seed in the right TPJ (for BUA). Each of these analyses will test the sensitivity of the attention networks to the hypothesised modulatory effects of subsequent memory status. Ultimately, this will show whether the functional connectivity of these attention-related seed regions is sensitive to memory encoding. Finally, we will reverse the previous question and explore the sensitivity of those regions displaying subsequent memory effects to the manipulation of attention as it might be the case that regions displaying subsequent memory effects may themselves be sensitive to attention.

\section{Materials \& methods}

\subsection{Subjects}

Twenty-seven subjects were recruited from the University of Surrey, and most participated to receive lab tokens. The project was advertised as a study into the neuroscience of visual attention, and subjects were not informed that this was a memory experiment. All subjects were aged between 18 and 36 years of age $(M=24.15, S D=5.57$, range $=18 ; 8$ male) and reported no known neurological or psychiatric conditions that might interfere with task performance. Written informed consent was acquired prior to experimentation, and all subjects verbally reported that they were unaware of the surprise recognition memory test during their debriefing. One subject was excluded due to excessive movements, and another due to scanner artefacts, resulting in a remaining sample of 25 subjects. The analysis of attention effects 
Table 1

Counts of stimuli allocated to each of the four encoding lists (search vs capture, target vs distracter), within each of the four blocks, according to their semantic categories.

\begin{tabular}{|c|c|c|c|c|}
\hline \multirow[b]{2}{*}{ Semantic Category } & \multicolumn{2}{|l|}{ Search } & \multicolumn{2}{|c|}{ Capture } \\
\hline & Target & Distractor $^{\mathrm{a}}$ & Target & Distractor $^{\mathrm{b}}$ \\
\hline \multicolumn{5}{|l|}{ In category (Reference) } \\
\hline Animals & 13 & 39 & 7 & 21 \\
\hline Foods & 13 & 39 & 7 & 21 \\
\hline Techs & 13 & 39 & 7 & 21 \\
\hline Tools & 13 & 39 & 7 & 21 \\
\hline \multicolumn{5}{|l|}{ Out category } \\
\hline Non-animals & 13 & 39 & 7 & 21 \\
\hline Non-foods & 13 & 39 & 7 & 21 \\
\hline Non-techs & 13 & 39 & 7 & 21 \\
\hline Non-tools & 13 & 39 & 7 & 21 \\
\hline TOTAL & 104 & 312 & 56 & 168 \\
\hline
\end{tabular}

a Distractors used during search trials always semantically opposed the search target. That is, they were from the out category when the target stimulus was from the in (i.e. reference) category and from the in category when the target stimulus was from the out category.

$\mathrm{b}$ Distractors used during Capture trials were congruent with the semantic category of the target stimulus.

included all 25 subjects; however, three further subjects were excluded from the SME analysis due to an insufficient number of trials associated with later forgetting, meaning that a comparison between subsequently remembered vs forgotten would have been too biased towards remembering.

\subsection{Stimulus organisation}

A total of 724 colour images of items on a plain white background, sampled from the Bank of Standardised Stimuli (BOSS) (Brodeur, Dionne-Dostie, Montreuil, \& Lepage, 2010; Brodeur, Guérard, \& Bouras, 2014), were presented to each participant throughout the study. Encoding stimuli were 160 targets and 480 distractors (totalling 640), organised into eight lists according to each semantic category (Table 1 ), where categories were organised in an (A, not A) design. Retrieval stimuli were 224 targets and distractors from the encoding phase (old), intermixed with an additional 84 unseen stimuli new.

The four reference semantic categories seen in Table 1 defined the task for each block, and subjects were given this information prior to starting each block, as this defined how to respond (in or out category; A, not A). For each of the four encoding blocks there were 26 search and 14 capture trials, with one half containing in- (reference category) and the other half containing out-category targets (see Fig. 1 for example trials). The use of unequal trial numbers for each condition was motivated by the notion that rare events capture attention in a reflexive manner (Han \& Marois, 2014; Kim, 2014; Posner, 2014; Sokolov, 1963). Stimuli from each semantic category were randomly assigned to the respective attention (search vs capture) and item (target vs distracter) conditions.

Due to the large total number of encoding stimuli (640), many were dropped for the retrieval phase. Since there were 56 capture trials (14 trials per block $\times 4$ blocks) in total, this was used as a reference point for the number of other stimuli to bring forward to retrieval. Thus, a random selection of 56 stimuli from each of the four attention-item conditions were brought forward to the retrieval phase and mixed with the 84 new stimuli. The 112 distractors that were retained in this way (56 from each attention condition) were randomly and uniformly selected from all possible positions (adjacent to, above, below, or diagonal) with respect to the target.

Finally, the assignment of stimuli to all conditions - as well as the response-button mapping - was counterbalanced across two versions of the experiment. This procedure ensured that no stimulus shared the same experimental condition across the two versions. All 724 stimuli were used in both versions.

\subsection{Procedure}

Subjects performed a semantic categorisation task inside the scanner and were instructed to indicate whether a target stimulus on each trial belonged to the reference category for that block or not (A, not A, see below for details). By performing this task, subjects were also incidentally encoding stimuli without being informed as such to ensure that motivation to encode did not confound the attention manipulation. The name of the reference category (see reference semantic categories in Table 1) was presented during the instructions prior to each block, and the block orders were randomised for each subject.

There were 160 encoding trials, where 104 (65\%) involved searching for the target stimulus (search condition) and 56 trials (35\%) involved a target that captured attention by quickly flickering off and then on again shortly after trial onset (see Fig. 1 for diagram). In both conditions, trials began with a $1000 \mathrm{~ms}$ fixation cross, followed by four images presented simultaneously (one target among three distractors) for $3000 \mathrm{~ms}$, followed by a jittered inter-trial interval (ITI, variable between $1000 \mathrm{~ms}$ and $6000 \mathrm{~ms}$, with a mean of $3000 \mathrm{~ms}$ ). In capture trials, $150 \mathrm{~ms}$ after stimulus onset, the target was removed for $150 \mathrm{~ms}$ (to ensure that the stimulus flicker occurred prior to eye movement), before returning for the remaining $2700 \mathrm{~ms}$. The reference semantic category was defined at the beginning of each block. On search trials, the target was defined as the single image which was not from the same semantic category as the remaining three (the target on half of the trials was from the reference category, on the other half it was not). For example, an in-category target for the animals block was an elephant, presented with three non-animal distractors: a guitar, a car, and a spray-bottle. This structure was reversed for the out-category trials, where the non-animal target was presented with three animal distractors. On capture trials, all four images were from the same semantic category, but the target was defined as the image that flickered off and then back on. Subjects were instructed to identify the target and to judge whether it was from the reference category ("in category") or not ("out category"). Both conditions were pseudorandomised such that a run of two consecutive capture trials was impossible, maintaining the perception of low frequency.

Following an approximately seven-minute retention period in which the anatomical scan was obtained, participants completed a surprise recognition memory task (reported elsewhere) for the remainder of the experiment.

\subsection{Functional MRI}

\subsubsection{Data acquisition}

Magnetic resonance imaging data were acquired on a 3-Tesla Siemens Magnetom Trio scanner at the Combined Universities Brain Imaging Centre (CUBIC), Royal University of London, UK. A total of eight functional scan runs (four at both encoding and retrieval) with 36 axial slices covering the whole brain were acquired with a $\mathrm{T} 2 \%$-sensitive multi-slice echo planar imaging (EPI) sequence (repetition time $=2.5 \mathrm{~s}$; echo time $=31 \mathrm{~ms}$; field of view $=30.48 \mathrm{~cm}$; slice order $=$ ascending interleaved; image matrix $=64 \times 64$; voxel size $=3 * 3 * 3 \mathrm{~mm} 3$; flip angle $=85^{\circ}$.

\subsubsection{Preprocessing \& analysis}

Data were preprocessed and later analysed using SPM12 (revision no. 7219, www.fil.ion.ucl.ac.uk/spm) (Friston, Ashburner, Kiebel, Nichols, \& Penny, 2007). All volumes were realigned (registered to the first image) and unwarped, slice time corrected, normalised to standard stereotactic space using the MNI template, and then smoothed using an $8 \mathrm{~mm}$ full-width half maximum Gaussian kernel. To ensure data quality, subjects were excluded from all analyses when movement exceeded 


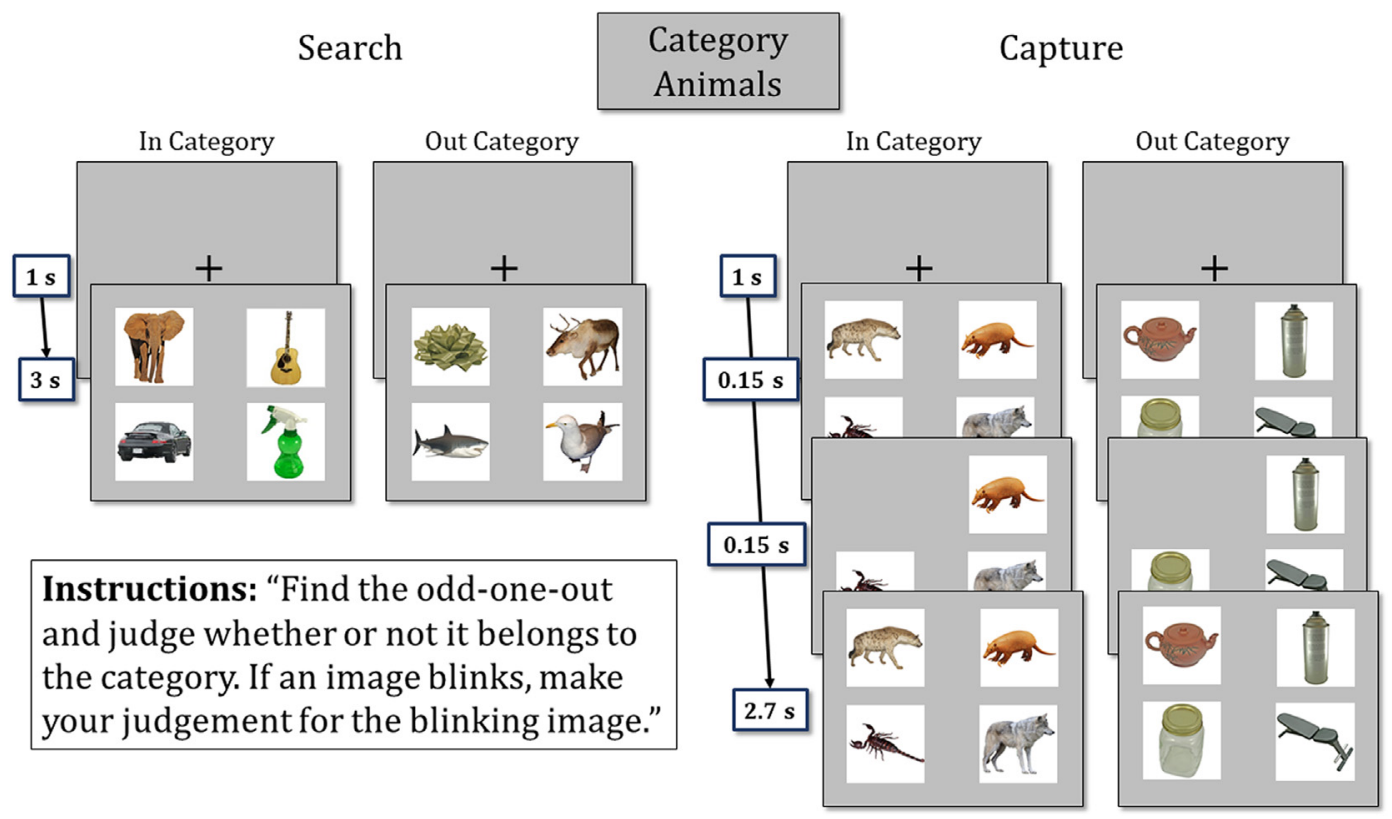

Fig. 1. Example in-category search (left side) and capture (right side) trials from the animals block. Prior to each block the reference semantic category was presented (e.g. "ANIMALS"), against which all target stimuli in the block should be judged.

$4.5 \mathrm{~mm}$ translation and/or $0.0375^{\circ}$ rotation, at any point throughout the experimental run. One subject was excluded on this basis. After preprocessing, two separate GLM analyses were created at the individual subject level for (1) attention at encoding, and (2) SMEs at encoding. Two GLMs were created because several search trials were not associated with a memory response as they were not included in the recognition phase and could therefore not be analysed for subsequent memory effects. Separating attention from subsequent memory in this way allowed for a full analysis of the attention effects. For all analyses, each behavioural event was convolved with a standard haemodynamic response function.

For the attention GLM analysis, search and capture trials were modelled as separate regressors. Due to the nature of the task, the exact time at which target encoding began in search trials was impossible to know. To account for this, for each participant and for each block, the median response time in capture trials (which, by definition, did not involve a period of sustained visual search) was subtracted from the median response time in search trials. This was used to estimate the search latency, allowing for a reasonable estimation of the onset of target encoding in search trials. Thus, onsets of search trials were corrected by adding the estimated search latency to the initial trial onset time.

For the SME GLM analysis, trials containing targets that were subsequently remembered (SR) and subsequently forgotten (SF) were modelled using four regressors: (1) search targets SR; (2) search targets SF; (3) capture targets SR; and (4) capture targets SF. Although confidence ratings were provided for each retrieval response, these were not accounted for in the SME analysis.

At the second level, a $2 \times 2$ within-subjects full-factorial ANOVA was used to test the effects of Attention (search, capture) and Subsequent Memory (SR, SF), as well as the Attention-by-SM interaction. This addresses the main question of whether attention impacts the neural correlates of subsequent memory. Furthermore, one-sample $t$ tests were used to follow up from the ANOVA with pairwise comparisons.

Psychophysiological interaction (PPI) analyses were carried out to test how functional connectivity of the attention networks differed according to subsequent memory. The DAN shows bilateral activation of the dPPC, while the VAN is mostly right lateralised (Vossel, Geng, \& Fink, 2014). For this reason, the seed regions were (1) the left dPPC; (2) the right dPPC; (3) the right TPJ. Additionally, five more seed regions were selected, based on the whole brain univariate subsequent memory analysis, for post hoc exploration. These were bilateral lateral occipital cortex (LOC), bilateral fusiform gyrus (FG), and right hippocampus, all of which displayed a positive SME. These were used to test whether positive SM regions show differential connectivity modulated by attention mode. Therefore, a total of eight PPI analyses were conducted.

For all analyses, an initial threshold of $\alpha=0.001$ uncorrected was used to identify clusters that were significant at $p_{\mathrm{FDR}}<0.05$ (for the GLM) or $p_{\text {FDR }}<0.00625$ (for the PPI, corrected for eight comparisons). Cluster-corrected maps were then projected onto a 1200 subject group average surface using the Human Connectome Project Workbench application (Washington University, St. Louis, MO, (Marcus et al., 2013)), and overlaid onto the template T1 image of Colin Holmes provided in the MRICron application (Rorden \& Brett, 2000).

\section{Results}

\subsection{Behaviour}

The results from the behavioural analysis are summarised in Table 2 and Fig. 2. Performance on the semantic categorisation task was not normally distributed, as shown in Fig. 2, with a ceiling effect indicating that participants were engaging with the task as instructed. Although one subject performed poorly on capture trials, they were not excluded

Table 2

Behavioural results split by attention condition. Table shows performance on the semantic categorisation task (Proportion Correct) and the probability of subsequent recognition (Probability SR). Response times are shown for all trials, as well as for the subset of stimuli carried forward into the recognition phase, for both subsequently remembered (SR) and subsequently forgotten (SF). Numbers in parentheses denote standard error of the mean.

\begin{tabular}{lll}
\hline & Search & Capture \\
\hline Proportion Correct & $0.85(0.01)$ & $0.91(0.02)$ \\
Probability SR & $0.70(0.02)$ & $0.73(0.02)$ \\
RT & $2965(98)$ & $2191(110)$ \\
RT (SR) & $2988(98)$ & $2211(114)$ \\
RT (SF) & $2928(106)$ & $2171(115)$ \\
\hline
\end{tabular}



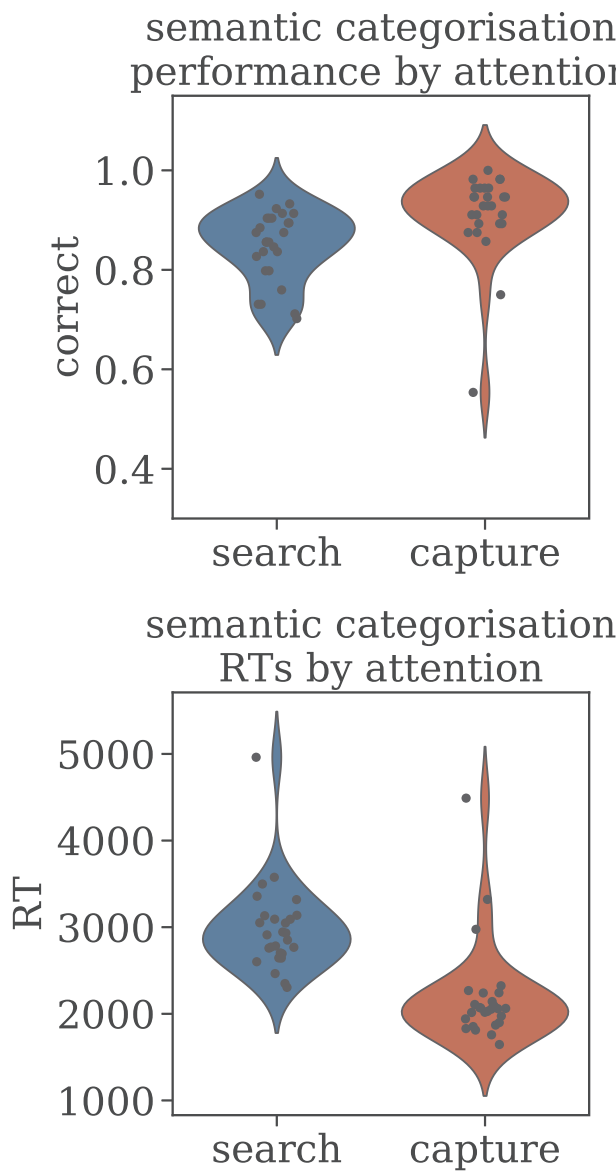

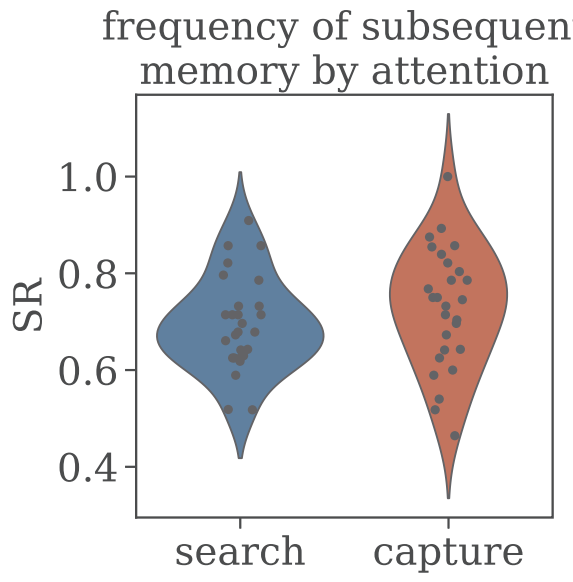

RTs by subsequent memory \& attention

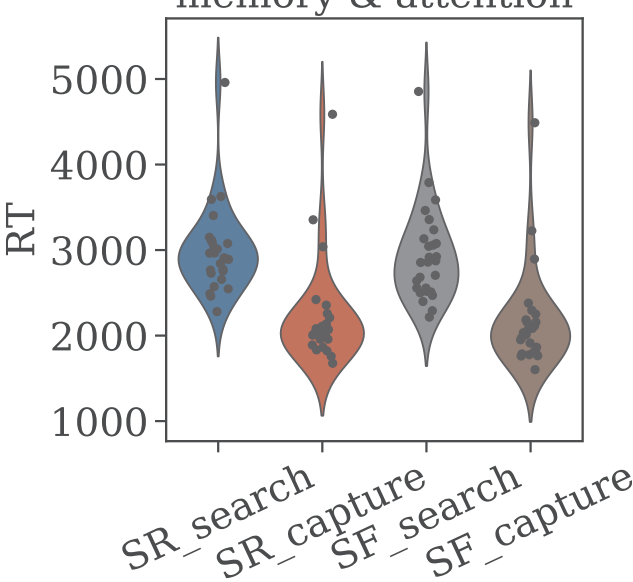

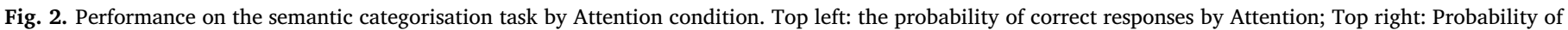

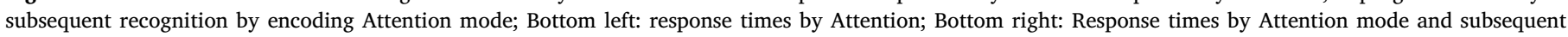
memory (SR, remembered; SF, forgotten).

as they performed normally in the retrieval phase. This showed that they were engaging correctly overall, and their poor performance on these trials was not taken to reflect a lack of target encoding. A Wilcoxon Signed Ranks test showed that semantic categorisation performance was significantly better for capture trials than for search trials $(W=41, z=4.54, p<.01, r=0.874)$.

Median response times were analysed using a $2 \times 2$ repeated measures ANOVA with factors attention (search, capture) and subsequent memory (SR, SF). Although the residuals were not normally distributed (positive skew), ANOVA is the only option for this analysis. One subject was excluded due to there being no subsequently forgotten capture trials (i.e. they remembered them all). This analysis showed a significant main effect of attention $(F(1,25)=189.384, p<.001$, $\eta_{p}^{2}=0.883$ ), indicating that response times were, unsurprisingly, slower on search trials compared with capture trials. In addition, a trending but non-significant main effect of subsequent memory $(F(1$, $25)=3.299, p=0.081, \eta_{p}^{2}=0.117$ ), and a non-significant interaction effect were observed.

Finally, the frequency of subsequent recognition success between search $(M=0.70, S D=0.10)$ and capture $(M=0.73, S D=0.12)$ conditions was analysed, showing a non-significant difference between the two conditions $(t(26)=1.773, p=.088$, Cohen's $d=0.30)$.

\subsection{Univariate $f M R I$ analyses}

3.2.1. Dorsal and ventral attention systems support attention search and capture

The search $>$ capture contrast revealed several clusters distributed across visual, dorsal attention, and frontoparietal control regions (blue, Fig. 3). The largest cluster extended bilaterally across middle occipital cortex, ventrally through the lingual gyrus and into the fusiform gyrus, and dorsally into the intraparietal sulcus and dorsal PPC of both hemispheres. Activity was also observed in bilateral superior frontal gyrus/frontal eye fields and supplementary motor areas. Finally, clusters were present in the region of the right anterior insula as well as bilateral putamen/caudate nucleus.

The capture $>$ search contrast (red, Fig. 3) revealed bilateral activation of the temporoparietal junction extending ventrally and laterally into the temporal lobe and dorsally into the ventral PPC. Furthermore, there was significant activation of the right middle frontal gyrus and bilateral inferior frontal gyrus/frontal opercula. Finally, significant clusters were present in the middle/posterior cingulate cortex and caudally in the anterior cingulate extending dorsally into the dorsomedial prefrontal cortex. Please refer to Table 3 for details of these comparisons.

\subsubsection{Attention mode did not interact with subsequent memory to influence} $B O L D$

The two-way ANOVA did not show a significant interaction between attention and subsequent memory. However, significant main effects 


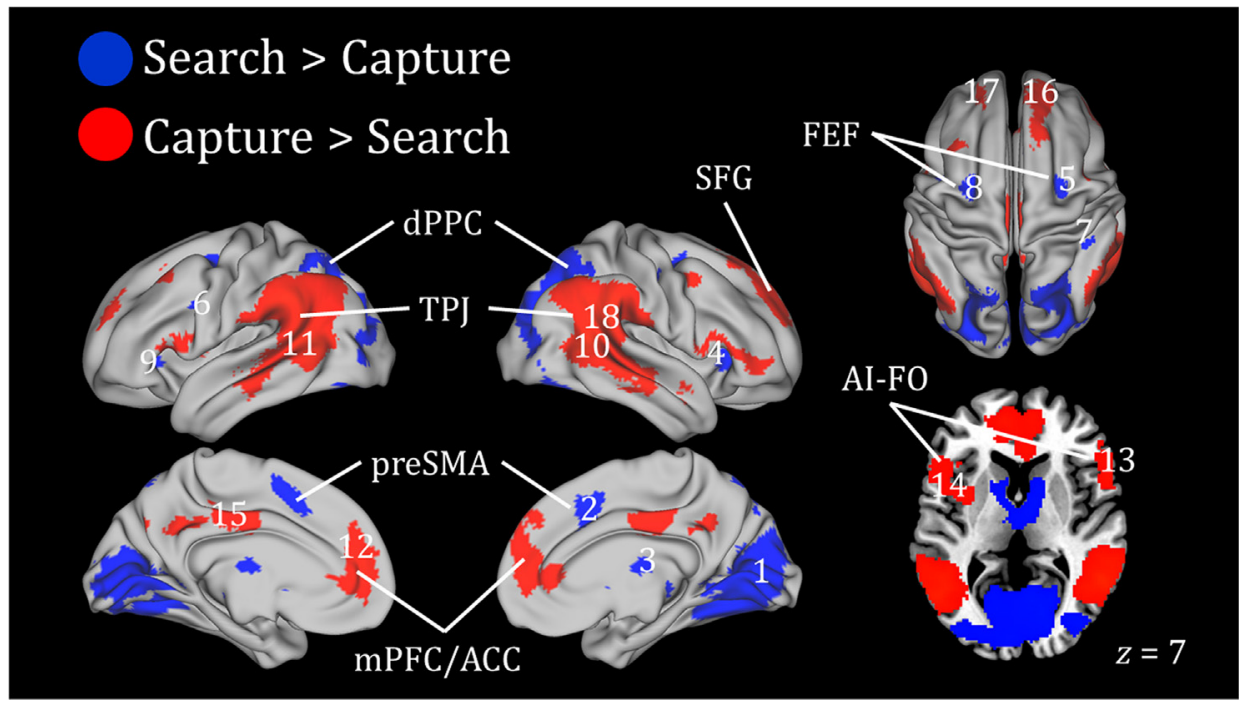

Fig. 3. Brain regions more associated with attention Search (blue) and those more associated with attention Capture (red). dPPC: dorsal posterior parietal cortex; SFG: superior frontal gyrus; AI-FO: anterior insula-frontal operculum; preSMA: presupplementary motor area; FEF: frontal eye fields; mPFC: medial prefrontal cortex; ACC: anterior cingulate cortex. (For interpretation of the references to colour in this figure legend, the reader is referred to the web version of this article.)

Table 3

Results of a whole-brain GLM analysis $(n=25)$ comparing attention search and capture. All clusters are significant at $p_{\mathrm{FDR}}<0.05$.

\begin{tabular}{|c|c|c|c|c|c|c|}
\hline Contrast & Region & $k$ & $T$ & $x$ & $y$ & $z$ \\
\hline \multicolumn{7}{|c|}{ search $>$ capture $(k \geq 70)$} \\
\hline 1 & Right calcarine cortex & 15,078 & 8.34 & 16 & -72 & 4 \\
\hline 2 & $\begin{array}{l}\text { Right supplementary motor } \\
\text { cortex }\end{array}$ & 1033 & 7.56 & 4 & 14 & 48 \\
\hline 3 & Right ventral diencephalon & 1831 & 7.04 & 6 & -28 & 0 \\
\hline 4 & Right anterior insula & 240 & 6.38 & 28 & 28 & -2 \\
\hline 5 & Right middle frontal gyrus & 404 & 6.28 & 26 & 0 & 52 \\
\hline 6 & Left precentral gyrus & 237 & 6.08 & -34 & 8 & 26 \\
\hline 7 & Right supramarginal gyrus & 102 & 5.55 & 44 & -36 & 44 \\
\hline 8 & Left superior frontal gyrus & 361 & 5.43 & -22 & -4 & 50 \\
\hline $\begin{array}{l}9 \\
\text { capture }>\end{array}$ & $\begin{array}{l}\text { Left orbital inferior frontal gyrus } \\
\text { search }(k \geq 170)\end{array}$ & 70 & 4.51 & -28 & 28 & -2 \\
\hline 10 & Right middle temporal gyrus & 6520 & 12.62 & 54 & -52 & 8 \\
\hline 11 & Left middle temporal gyrus & 6256 & 10.66 & -52 & -60 & 10 \\
\hline 12 & $\begin{array}{l}\text { Left superior frontal gyrus } \\
\text { medial segment }\end{array}$ & 4334 & 7.28 & -8 & 50 & 6 \\
\hline 13 & $\begin{array}{l}\text { Right opercular inferior frontal } \\
\text { gyrus }\end{array}$ & 888 & 6.71 & 60 & 22 & 10 \\
\hline 14 & Left anterior insula & 699 & 6.45 & -34 & 4 & 6 \\
\hline 15 & Left posterior cingulate gyrus & 2255 & 6.36 & -10 & -32 & 40 \\
\hline 16 & Right middle frontal gyrus & 210 & 6.32 & 44 & 6 & 44 \\
\hline 17 & Left middle frontal gyrus & 328 & 5.46 & -34 & 28 & 46 \\
\hline 18 & Right superior temporal gyrus & 170 & 5.33 & 54 & 4 & -16 \\
\hline
\end{tabular}

were found for both factors (see Table 4). We describe these attention effects from the attention GLM itself (see Section 3.2.1), as the subsequent memory GLM on which this ANOVA was based did not contain the search trials that were discarded for the later recognition task (see Section 2.4.2. above).

\subsubsection{Subsequent memory effects in regions sensitive to object processing}

The SR > SF contrast (Fig. 4, green) showed significant changes in BOLD responses in bilateral lateral occipital cortex, bilateral fusiform gyrus, and right hippocampus. On the other hand, the SF > SR contrast (Fig. 4, orange) showed significant clusters in bilateral superior frontal sulci/frontal eye fields only. These results are presented in Table 5.

\subsection{Psychophysiological interaction analyses of task-based functional connectivity}

Despite there being no significant attention $\times$ subsequent memory interaction effect, patterns of functional connectivity associated with
Table 4

Results of a $2 \times 2$ Full-factorial ANOVA $(n=22)$ with Attention (search vs. capture) and subsequent memory (SR vs. SF) as within-subjects factors. All clusters reported are significant at $p_{\mathrm{FDR}}<0.05$ with cluster thresholds $(k)$ reported for each effect. $\mathrm{ME}=$ main effect; $\mathrm{SM}=$ subsequent memory.

\begin{tabular}{|c|c|c|c|c|c|c|}
\hline Contrast & Region & $k$ & $F$ & $x$ & $y$ & $z$ \\
\hline \multicolumn{7}{|c|}{ ME Attention $(k \geq 132)$} \\
\hline 1 & Left middle temporal gyrus & 4729 & 80.01 & -48 & -54 & 10 \\
\hline 2 & Right middle temporal gyrus & 5798 & 76.12 & 56 & -54 & 8 \\
\hline 3 & Right fusiform gyrus & 14,138 & 62.95 & 28 & -50 & -14 \\
\hline 4 & Right anterior insula & 234 & 36.89 & 30 & 26 & -2 \\
\hline 5 & $\begin{array}{l}\text { Right supplementary motor } \\
\text { cortex }\end{array}$ & 535 & 32.01 & 4 & 16 & 48 \\
\hline 6 & $\begin{array}{l}\text { Right superior frontal gyrus } \\
\text { medial segment }\end{array}$ & 2349 & 30.99 & 2 & 52 & 18 \\
\hline 7 & Left middle frontal gyrus & 365 & 30.42 & -34 & 22 & 48 \\
\hline 8 & $\begin{array}{l}\text { Right triangular part of the } \\
\text { inferior frontal gyrus }\end{array}$ & 445 & 28.76 & 52 & 36 & 2 \\
\hline 9 & Left posterior cingulate gyrus & 1674 & 27.74 & -4 & -40 & 38 \\
\hline 10 & Right Pallidum & 165 & 24.97 & 10 & 4 & 0 \\
\hline 11 & Right middle frontal gyrus & 132 & 24.72 & 28 & 0 & 52 \\
\hline \multicolumn{7}{|c|}{$\operatorname{ME~SM~}(k \geq 147)$} \\
\hline 12 & Right fusiform gyrus & 2277 & 43.48 & 28 & -52 & -12 \\
\hline 13 & Left lingual gyrus & 1916 & 29.21 & -24 & -46 & -8 \\
\hline 14 & Right superior frontal gyrus & 503 & 27.44 & 22 & 14 & 54 \\
\hline 15 & Left middle frontal gyrus & 193 & 26.61 & -24 & 22 & 44 \\
\hline 16 & Right Ventral diencephalon & 147 & 23.72 & 26 & -24 & -8 \\
\hline 17 & Left precuneus & 210 & 22.78 & -18 & -60 & 22 \\
\hline 18 & Left precuneus & 150 & 22.07 & -6 & -50 & 44 \\
\hline 19 & Right supramarginal gyrus & 170 & 20.18 & 60 & -38 & 38 \\
\hline
\end{tabular}

regions of interest may be modulated by the experimental variables. While the standard univariate analysis reveals all brain voxels that show significant changes in blood flow induced by the task, it considers each voxel independently. Psychophysiological interaction (PPI) analysis on the other hand looks at the association between activity in a seed region of interest and all other voxels in the brain (the physiological variable) and asks how these associations change according to the task (the psychological variable).

First, we carried out three PPI analyses with seed regions placed in the right and left dPPC, and the right TPJ. These regions were selected, a priori, based on previous research showing these regions to be key nodes of the dorsal and ventral attention networks, respectively. Furthermore, they showed the expected task-related activations in the present study. We then carried out five post hoc exploratory PPI analyses to test if the functional connectivity of regions displaying positive subsequent memory effects was modulated by attention. 


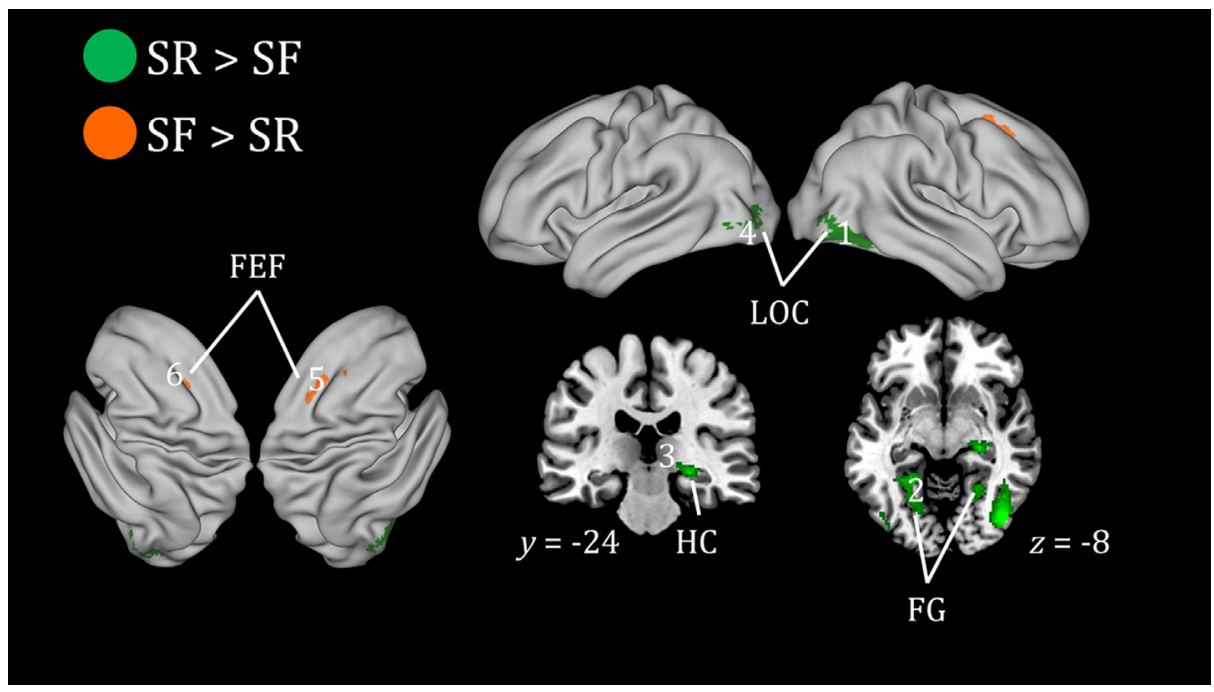

Fig. 4. Brain regions showing activity that was predictive of later recognition (green; subsequently remembered, SR) and later forgetting (orange; subsequently forgotten, SF). FEF: frontal eye fields; LOC: lateral occipital cortex; FG: fusiform gyrus; HC: hippocampus. (For interpretation of the references to colour in this figure legend, the reader is referred to the web version of this article.)

Table 5

Results of a whole-brain GLM analysis comparing items at encoding that were subsequently remembered (SR) with those that were subsequently forgotten (SF). All clusters are significant at $p_{\mathrm{FDR}}<0.05$.

\begin{tabular}{lllllll}
\hline Contrast & Region & $k$ & $T$ & $x$ & $y$ & $z$ \\
\hline SR $>$ SF $(k \geq 137)$ & & & & & \\
1 & $\quad$ Right lateral occipital cortex & 864 & 7.19 & 42 & -74 & -8 \\
2 & Left lingual gyrus & 265 & 5.92 & -24 & -48 & -10 \\
3 & Right hippocampus & 137 & 5.53 & 26 & -24 & -8 \\
4 & Left lateral occipital cortex & 314 & 4.82 & -32 & -90 & 6 \\
SF $>$ SR $(k \geq 136)$ & & & & & \\
5 & Right superior frontal gyrus & 335 & 6.34 & 20 & 8 & 54 \\
6 & Left superior frontal gyrus & 136 & 5.72 & -28 & 18 & 40 \\
\hline
\end{tabular}

Table 6

Results from the PPI analyses for the key nodes of the two attention networks according to subsequent memory.

\begin{tabular}{|c|c|c|c|c|c|c|c|}
\hline PPI Contrast & Seed & Region & $k$ & $T$ & $x$ & $y$ & $z$ \\
\hline \multicolumn{8}{|c|}{ Search SF $>$ Search SR } \\
\hline \multicolumn{8}{|c|}{ Right dPPC $(k \geq 270)$} \\
\hline & 1 & Left calcarine cortex & 4039 & 6.17 & -16 & -88 & 10 \\
\hline & 2 & $\begin{array}{l}\text { Right superior parietal } \\
\text { lobule }\end{array}$ & 973 & 4.88 & 22 & -60 & 48 \\
\hline & 3 & $\begin{array}{l}\text { Left supplementary motor } \\
\text { cortex }\end{array}$ & 270 & 4.70 & -8 & 4 & 58 \\
\hline \multicolumn{8}{|c|}{ Left dPPC $(k \geq 171)$} \\
\hline & 4 & Left lingual gyrus & 1216 & 6.52 & 0 & -82 & 2 \\
\hline & 5 & Left middle frontal gyrus & 172 & 5.70 & -32 & 12 & 36 \\
\hline & 6 & Left supramarginal gyrus & 181 & 4.80 & -64 & -34 & 34 \\
\hline & 7 & $\begin{array}{l}\text { Right superior parietal } \\
\text { lobule }\end{array}$ & 572 & 4.76 & 20 & -64 & 48 \\
\hline & 8 & Right supramarginal gyrus & 171 & 4.51 & 40 & -40 & 40 \\
\hline \multicolumn{8}{|c|}{$\begin{aligned} \text { Capture SR }> & \text { Capture SF } \\
& \text { *Right TPJ }(k \geq 717)\end{aligned}$} \\
\hline & 9 & $\begin{array}{l}\text { Left posterior cingulate } \\
\text { gyrus }\end{array}$ & 717 & 4.62 & -6 & -26 & 22 \\
\hline
\end{tabular}

* Initial threshold relaxed to 0.01 ; did not survive correction for multiple comparisons.

3.3.1. Sensitivity of dorsal parietal cortex connectivity to subsequent memory

With a seed defined in the right dPPC ( $\left.x y z=\left[\begin{array}{lll}30 & -50 & 48\end{array}\right]\right)$, we tested whether functional connectivity differed - on search trials between successful and unsuccessful encoding. We found that encoding failure relative to success was associated with increased dPPC connectivity with precuneus, ventral visual regions, and the SMA/preSMA.
The ventral visual cluster extended rostrally into posterior fusiform cortex with larger effects in the left hemisphere. The cluster in the SMA $\left(p_{\mathrm{FDR}}=0.02\right)$ did not survive correction for the total number of PPI analyses carried out $\left(\alpha_{\mathrm{FDR}}=0.00625\right)$. Since the dorsal attention network is largely bilateral, the same analysis was carried out with a seed in the left dPPC $(x y z=[-26-5654])$, revealing a similar pattern of results: increased functional connectivity with a cluster of the ventral occipital cortex (again extending into fusiform), along with the right dPPC (see Fig. 5, top, and Table 6 for these results).

\subsubsection{Sensitivity of right temporoparietal junction connectivity to subsequent memory}

A seed in the right TPJ ( $\left.x y z=\left[\begin{array}{lll}64 & -40 & 24\end{array}\right]\right)$ was used to test whether connectivity differed - on capture trials - between encoding success and failure (see Table 6). With the same initial statistical threshold as above ( $\alpha=0.001)$, there was no effect. However, relaxing the initial threshold $(\alpha=0.01)$ showed an increase in coupling with the PCC during encoding success relative to failure $\left(p_{\mathrm{FDR}}=0.018\right)$. This effect did not survive correction for multiple analyses but is nevertheless reported as it may be of interest for future studies.

\subsubsection{Sensitivity of subsequent memory effects to attention mode}

The group level SR > SF GLM results were used to specify five further seed regions for post hoc exploratory PPI analyses of how their functional connectivity differs according to attention mode. These were the left and right lateral occipital cortex (LOC; xyz left $=\left[\begin{array}{l}-32 \\ -90\end{array}\right]$, $x y z$ right $=[42-74-8]$ ), the left and right fusiform gyrus (FG; $x y z$ left $=\left[\begin{array}{lll}-24 & -48-10\end{array}\right]$, xyz right $=\left[\begin{array}{lll}44 & -58 & -16\end{array}\right]$, , and the right hippocampus $(x y z=[26-24-8])$. The LOC and FG regions were chosen because they are associated with visual object processing (Bracci et al., 2017; Goddard, 2017; Grill-Spector \& Weiner, 2014) and the encoding stimuli used in this study were pictorial objects; they have also been shown to display positive SMEs. The hippocampus was additionally chosen due to its well-known involvement in episodic encoding, and in this case only the right hippocampus showed a significant positive SME.

Only the LOC regions showed effects that reached significance. On successfully encoded trials there was an increase in connectivity between left LOC and right FG when comparing search with capture, along with a similar increase between right LOC and bilateral FG connectivity for the same comparison (see Fig. 5, bottom, and Table 7). This suggests that the LOC is a region that not only displays positive subsequent memory effects, but that these effects are accompanied by patterns of functional connectivity with ventral visual regions that are sensitive to attention. 


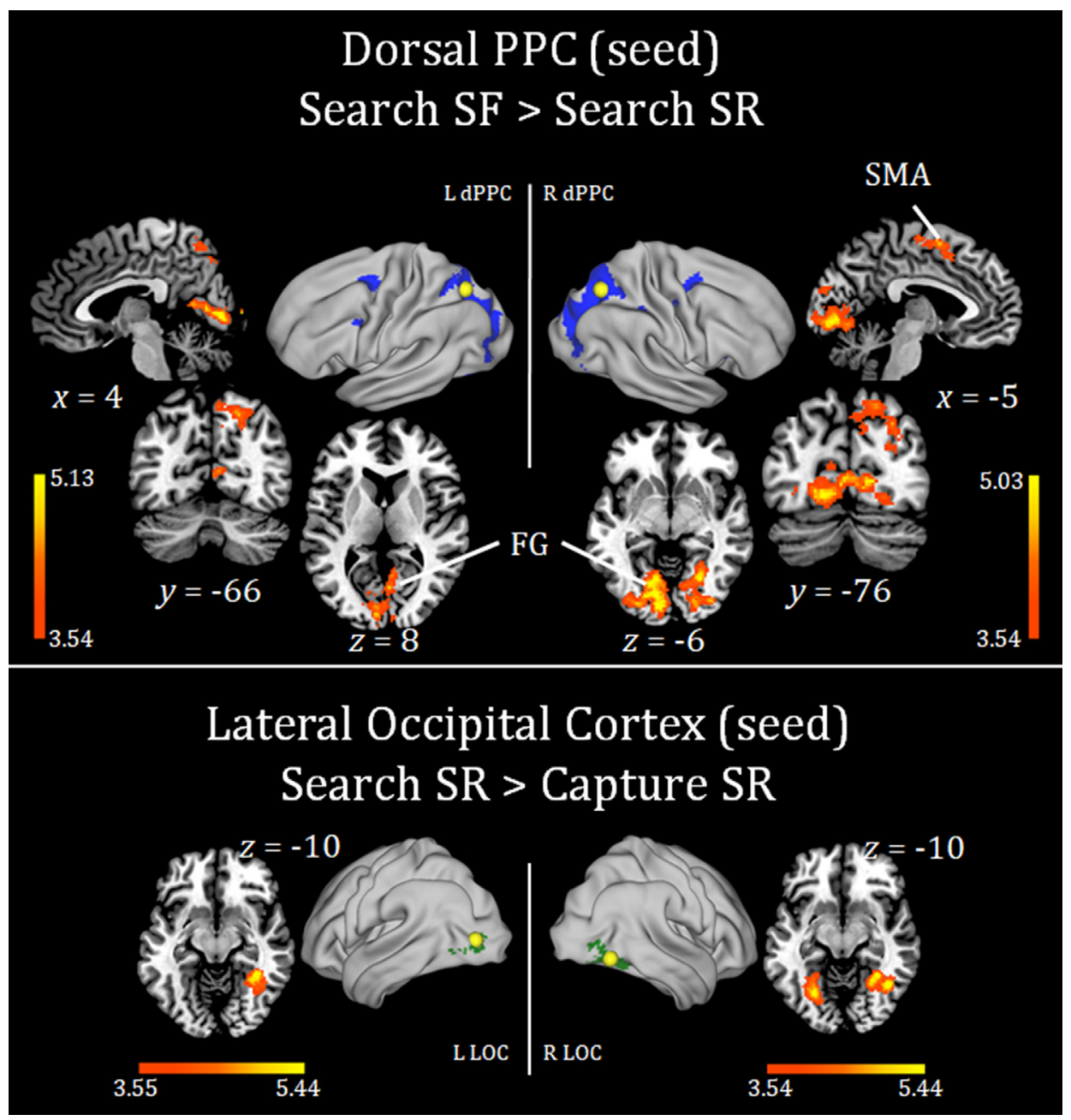

Fig. 5. Top left: From the left dPPC seed, activation of the precuneus and FG for subsequently forgotten > subsequently remembered search trials. Top right: From the right APPC seed, activation of the FG and SMA for subsequently forgotten > subsequently remembered search trials. Bottom left: From the left LOC seed, activation of the right FG for search > capture subsequently remembered trials. Bottom right: From the right LOC, activation of bilateral FG for search > capture subsequently remembered trials.

Table 7

Regions displaying subsequent memory effects in the univariate analysis that showed significant changes in functional connectivity according to attention mode.

\begin{tabular}{|c|c|c|c|c|c|c|c|}
\hline PPI Contrast & Seed & Region & $k$ & $T$ & $x$ & $y$ & $z$ \\
\hline \multicolumn{8}{|c|}{ Search SR > Capture SR } \\
\hline \multicolumn{8}{|c|}{ Right LOC $(k \geq 147)$} \\
\hline & 1 & $\begin{array}{l}\text { Right fusiform } \\
\text { gyrus }\end{array}$ & 693 & 6.25 & 42 & -58 & -10 \\
\hline & 2 & Left lingual gyrus & 426 & 5.83 & -22 & -64 & -6 \\
\hline & 3 & $\begin{array}{l}\text { Left middle } \\
\text { occipital gyrus }\end{array}$ & 147 & 4.25 & -32 & -84 & 14 \\
\hline \multicolumn{8}{|c|}{ Left LOC $(k \geq 359)$} \\
\hline & 4 & $\begin{array}{l}\text { Right fusiform } \\
\text { gyrus }\end{array}$ & 359 & 6.67 & 36 & -44 & -10 \\
\hline
\end{tabular}

\section{Discussion}

The goal of the present study was to test how manipulations of attention mode influence subsequent recognition memory and its neural correlates at encoding. We introduced a novel task that differentiated top-down attention (TDA; search) from bottom-up attention (BUA; capture). An advantage of this task was that it enabled such a manipulation while using meaningful pictorial stimuli for memory encoding.

First, a comparison was made between the BOLD response on search and capture trials, testing for a difference in the neural correlates of these two forms of attention. It was found that visually searching among competing stimuli for a target elicited increased activation of the DAN and a subset of regions of the frontoparietal control network (FPCN: bilateral IPS, preSMA). On the other hand, attentional capture by the abrupt stimulus flicker elicited increased activity in the VAN, along with the cingulo-opercular salience network (anterior insula/ frontal operculum, ACC). We then compared trials that were subsequently remembered with those that were subsequently forgotten and tested whether these effects differed according to the form of attention by which they were encoded. Bilateral LOC and FG, along with the right hippocampus, showed positive SMEs, while bilateral FEF showed negative SMEs. Attention mode did not significantly interact with encoding to produce differences in either the behavioural or whole-brain univariate fMRI data. Finally, we carried out psychophysiological interaction (PPI) analyses to test how functional connectivity patterns relating to the two attention networks might be impacted by subsequent memory, as well as how regions sensitive to subsequent memory might be impacted by attention. On search trials, the dorsal posterior parietal cortex (dPPC) showed greater coupling with bilateral fusiform cortex during unsuccessful encoding relative to successful encoding; on capture trials the temporoparietal junction (TPJ) showed no significant modulation of functional connectivity according to subsequent memory. Post hoc exploration of functional connectivity associated 
with the LOC revealed increased coupling with FG on search relative to capture trials during successful encoding.

\subsection{Attention effects}

The manipulation between TDA and BUA in the present study elicited clear responses within the DAN during search trials, and clear responses in the VAN during capture trials, showing that our manipulation was successful and in line with Corbetta and Shulman's dual attention model (Corbetta et al., 2008; Corbetta \& Shulman, 2002). Thus, the data presented here show further support for the involvement of the DAN and VAN in TDA and BUA, respectively, and they also highlight the reliability of these effects by demonstrating them in a novel task. Additionally, the involvement of control networks across both conditions reflects the interdependence between cognitive control and attentional processes.

Search trials elicited increased BOLD signals in the posterior intraparietal sulcus (pIPS) and the pre-supplementary motor area (preSMA). This is compatible with the presumed function of these two regions of the FPCN: the pIPS contributes to the initiation of a task-set (Dosenbach et al., 2006; Marek \& Dosenbach, 2018) and the preSMA to response monitoring and inhibition (Cieslik, Mueller, Eickhoff, Langner, \& Eickhoff, 2015; Mostofsky \& Simmonds, 2008). This effect is not surprising because search trials required the identification of and comparison between competing stimuli within the stimulus array, as well as the concurrent planning of a response. This was not a feature of capture trials, given that the target was always apparent then, eliminating the need for target-distractor discrimination, and reducing the concurrent demand for joint discrimination and motor planning. Despite displaying right anterior insula activation, there was no observed coactivation of the ACC, suggesting that the cingulo-opercular "salience" network (CON) was less involved on search trials.

Conversely, capture trials also produced activations in the left insula, bilateral frontal opercula, and the ACC-key regions of the CON (Dosenbach, Fair, Cohen, Schlaggar, \& Petersen, 2008; Dosenbach et al., 2007; Menon \& Uddin, 2010; Seeley et al., 2007), which overlaps with - or can even be considered a part of - the VAN (Kucyi, Hodaie, \& Davis, 2012; Yeo et al., 2011). The insular cortex is functionally and structurally heterogenous (Evrard, 2018; Kurth, Zilles, Fox, Laird, \& Eickhoff, 2010; Uddin, Nomi, Hébert-Seropian, Ghaziri, \& Boucher, 2017); however, it seems likely that this region not only integrates visceral bodily signals with cognitive processes (Uddin, 2015; Uddin et al., 2017), but that it also contributes generally to performance monitoring, focused attention, and arousal/alerting (Nelson et al., 2010; Uddin, 2015), potentially even driving the switching between the default mode network (DMN) and FPCN (Uddin, 2015; Zhou et al., 2018). The ACC has similarly been associated with a broad range of functions, but the current view is that it is involved in integrating prediction error signals with high-level motor intentions, ultimately driving response outputs in the service of resolving conflicts between expectations and observations (Ham, Leff, de Boissezon, Joffe, \& Sharp, 2013; Holroyd \& Umemoto, 2016). Together, these two structures cooperatively enable the identification of behaviourally/homeostatically relevant stimuli among competing internal and external inputs, and integrate cognition with appropriate motor outputs (Uddin et al., 2017). In the present context, changes in arousal may be induced by the detection of salience (insula/frontal opercula), which is likely to cooccur with the detection of conflict due to expectancy violation (ACC), requiring an updating of the task set (Dosenbach et al., 2007; Ham et al., 2013; Han, Eaton, \& Marois, 2019; Holroyd et al., 2004; Menon \& Uddin, 2010). This neatly reflects the updating of behavioural goals following attention capture in the present task. Prior to each trial, the goal was typically to search for the target, given that $65 \%$ of trials required this. When the target blinked shortly after the trial onset, however, this required a shift in goal state from search initiation to object processing and semantic categorisation, following the registering of a behaviourally salient event. This is a process that is likely supported by the CON (Holroyd et al., 2004; Ridderinkhof, Ullsperger, Crone, \& Nieuwenhuis, 2004).

It is notable that the regions associated with the BUA capture trials appear to involve a composite of the VAN, CON, and the DMN. First, the TPJ cluster extends diffusely within the vicinity of both the DMN (Buckner \& DiNicola, 2019) and the VAN (Corbetta et al., 2008; Corbetta \& Shulman, 2002), highlighting the known anatomical similarities between these networks (see Yeo et al., 2011). However, based on the a priori hypothesis that this region would be involved in BUA, and previous findings associating the more anterior aspect of the TPJ (the location of the peak activity in the present study) with the VAN (Igelström \& Graziano, 2017), the current TPJ activity is taken to index the involvement of the VAN. In a similar way to how the TPJ spans both the VAN and DMN, both the rostral ACC and the frontal operculum overlap with the DMN and the VAN/CON. Given that the BUA condition was expected to involve a higher degree of salience processing (aligning with the expected functions of the $\mathrm{CON}$ ), and given that the task required an active rather than a passive state, we interpret the effects in the rostral ACC and frontal operculum as relating to the active VAN/ CON rather than the passive DMN. Maintaining the ability to rapidly switch from a default passive to an active state is likely an important survival function, and there is evidence that this switching is driven by the salience detection mechanism of the CON/VAN (Goulden et al., 2014; Zhou et al., 2018). Therefore, the overlap seen between these networks is highly plausible. The main conclusions to be drawn from this are that the DAN coordinated with the FPCN during TDA, while the VAN coordinated with the CON during BUA trials. Given that the CON is linked to cognitive control (Menon \& Uddin, 2010; Uddin, 2015), this clearly highlights the close functional associations between networks supporting attention (the DAN and VAN) and those supporting cognitive control (the FPCN and CON).

\subsection{No interaction between subsequent memory and attention}

Both the behavioural and the neuroimaging analyses gave no evidence for a modulatory influence of attention mode on subsequent memory. The frequency of successful subsequent recognition did not differ between stimuli encoded via attention search or capture. Moreover, the neuroimaging GLM analysis showed that subsequent memory effects were also not significantly affected by attention mode. Relaxing the $\alpha$ threshold for brain activation to improve statistical power gave no suggestion of a Type II error in this case - there was still no observable effect.

As discussed above, the attention manipulation was a success, but the semantic categorisation task may have influenced the outcome. Specifically, upon identification of the target, the required semantic judgement demands were held constant across trials in both conditions, and in order to make this judgement, some degree of elaborative semantic processing was required which might have overshadowed any potential influence of attention mode on encoding (additional implications of this are discussed below). Indeed, it is well established by the levels of processing effect that attending to semantic characteristics leads to better encoding compared with processing of shallow structural features (Craik, 2002; Craik \& Lockhart, 1972). If this is the case, then it would mean that any direct influence of attention on encoding is likely very small and is easily superseded by the depth of subsequent processing. This is a consideration that cannot be ruled out with the current data, so it would be necessary to address this in future work where the demand of the semantic judgement is either simplified or systematically varied. Furthermore, the strong attention effect combined with the lack of a significant interaction with subsequent memory, in the context of the present study, could imply that the SMEs observed are quite robust. However, this is tentative given that it is based on a null finding, and the above suggestion to vary the demands of the semantic judgement task would be a suitable way to explicitly test this. 
What is clear is that attention mode did not appear to significantly impact the behavioural effects or the whole-brain univariate activation patterns relating to encoding success. This also means that, unlike previous work (Uncapher et al., 2011), we found no evidence that BUA results in poorer subsequent recognition. This is somewhat unsurprising, as there has not been extensive direct research to show what effect should be predicted. As mentioned in the introduction, the cueing paradigm may confound BUA with conflict processing, which would explain why the cost to memory from BUA was not observed in the current task, although further research is needed to test this hypothesis. Nevertheless, it appears that BUA does not necessarily impair encoding, and that TDA does not necessarily improve it.

\subsection{Positive \& negative subsequent memory}

We found positive SMEs in bilateral LOC and FG, along with right hippocampus, in line with prior work (Kim, 2011; Paller \& Wagner, 2002; Uncapher \& Rugg, 2009). The LOC and FG regions form part of the ventral visual stream, with a well-established role in object processing and categorisation (Bracci et al., 2017; Goddard, 2017; GrillSpector \& Weiner, 2014), and strong associations with the medial temporal lobe system (Connor \& Knierim, 2017). This is a complex network with a bidirectional structure that codes for increasing levels of abstraction in parallel, beginning with perceptual inputs to the primary visual cortex and eventually producing abstract representations of object identities in the anterior inferior (Kravitz, Saleem, Baker, Ungerleider, \& Mishkin, 2013) and medial temporal lobe (Connor \& Knierim, 2017).

From a networks perspective, the LOC is also clustered within the DAN (Gordon et al., 2016; Power et al., 2011; Yeo et al., 2011), reflecting the known links between those areas associated with top-down processing and those with visual object processing (Cloutman, 2013; Milner, 2017). For example, the DAN not only spans frontal and parietal cortices, but it also has significant between-network functional connectivity to sensory regions including visual cortex via area MT+, which sits at the intersection between the DAN and the visual network (Power et al., 2011; Yeo et al., 2011). It has therefore been proposed that this ventral posterior region of the DAN in particular may act as a bridge between attentional and visual functions (Yeo et al., 2011). This is in line with the view that parietal attentional mechanisms influence visual processing (see below with regards to the functional connectivity results) (Desimone \& Duncan, 1995; Kastner \& Ungerleider, 2001; Sengpiel \& Hübener, 1999; Ungerleider, 2000). In the present study, positive SMEs were observed in the LOC and FG, suggesting that successful encoding involved part of the DAN regardless of the attention manipulation. While it is likely that some base level of elaboration would be required to encode information in most cases (thereby eliciting a response in these regions), given that the task under both conditions directly involved semantic categorisation, it cannot be ruled out that semantic elaboration itself contributed to the effects observed since this was held constant across the two attention conditions. Nevertheless, these regions have been shown in meta analytic work to be associated with pictorial item encoding in general (Kim, 2011), and we therefore interpret these ventral visual positive SMEs effects as a reflection of such integration between top-down control (DAN mediated) and incoming visual processes (ventral visual) in the service of object identification, representation, and elaboration.

It is possible that the increased involvement of LOC and FG for subsequently remembered targets was the result of more efficient object recognition and internal item representation, reflecting an increased fit between the presented stimulus and internal criteria for semantic category membership (implying a potential role for top-down processes to influence these regions), leading to better subsequent memory. Furthermore, these subsequent memory effects along the ventral visual cortex could relate to downstream hippocampal pattern separation mechanisms: stronger internal representations of an attended stimulus increases the likelihood of arbitrary item-context bindings that are known to support more successful encoding (Opitz, 2010). The LOC is known to process object shape (Kourtzi \& Kanwisher, 2001; Malikovic et al., 2016), while fusiform regions processes both general object categories and specific object features (Burgund \& Marsolek, 1997; GrillSpector \& Weiner, 2014; Marsolek, 1999; Marsolek \& Burgund, 2008; McMenamin, Deason, Steele, Koutstaal, \& Marsolek, 2015; Rosenke et al., 2018). Importantly, when a stimulus is repeatedly presented, regions of fusiform cortex display greater response habituation (repetition suppression) (Koutstaal et al., 2001; Simons, Koutstaal, Prince, Wagner, \& Schacter, 2003), which is interpreted as reflecting a transition of information from novel to familiar, thereby indexing memory encoding (Kim, 2011, 2017). Further to this, the hippocampus is well known to display increased BOLD responses during successful encoding (Davachi, Mitchell, \& Wagner, 2003; H. Kim, 2011; Prince, Daselaar, \& Cabeza, 2005), which is thought to reflect the binding of information into durable memory representations (Diana, Yonelinas, \& Ranganath, 2007; Kirwan, Wixted, \& Squire, 2008; Opitz, 2010; Squire, Stark, \& Clark, 2004). These findings therefore indicate clear activation patterns reflecting the encoding of object-based information into memory.

On the other hand, we found negative SMEs in bilateral FEFs only. It should be noted that these were both more anterior to the clusters seen in the attention-based activation from the search > capture contrast, although they still fell within the FEF region of the DAN. We interpret this as a reflection of increased attentional shifts due to exploration of the stimulus display on each trial, where more exploration is likely to lead to less specific stimulus encoding, regardless of the attention condition. This is because the FEF is not typically linked to stimulus encoding, but rather to shifts of overt and covert attention (Posner, 2014; Thompson, Biscoe, \& Sato, 2005), which is a requirement for selecting stimuli for encoding. Aside from this, there were no other obvious negative subsequent memory effects. It has previously been suggested that these are either generally more elusive than positive effects, or that they are simply less frequently reported (Uncapher \& Wagner, 2009). Importantly, human recognition memory performance is well known to be strong, with research demonstrating highly accurate recognition performance for large numbers of encoded stimuli, often across extended periods of time (Shepard, 1967; Standing, 1973). This means that, relative to subsequently remembered items, there are typically far fewer subsequently forgotten trials. While this lower number of subsequently forgotten trials does not preclude the study of negative SMEs, it is important to consider that the power to detect negative SMEs may be inherently low, which likely explains why such effects are less commonly reported, and why they appear to be scarce in the present study.

Despite the range of encoding effects observed, it is worth noting that other subsequent memory effects seen in the literature were not apparent in the current data. That lateral prefrontal effects were not observed is possibly due to the stimulus material being pictorial as opposed to words, as a meta-analysis showed - in a direct comparison of pictorial and word stimuli - that pictorial stimuli show a relative absence of lateral prefrontal activation (Kim, 2011). Furthermore, the lack of PPC subsequent memory effects can be explained by a reportedly low base rate of approximately $40 \%$ of publications showing subsequent memory effects anywhere in the brain (Uncapher \& Wagner, 2009). It is unclear what factors might contribute to the replicability of such effects in the PPC.

\subsection{Functional connectivity}

Bilateral dorsal PPC and the right TPJ were selected as a priori seed regions for which PPI analyses were conducted. It was hypothesised that these regions would each increase coupling with ventral visual regions during successful encoding, for search (dorsal PPC) and capture (right TPJ), and that this would be an index of increased semantic processing of stimuli (Uncapher et al., 2011). However, our results did 
not support these hypotheses.

Although the TPJ seed showed no significant connectivity patterns according to subsequent memory, the dorsal PPC seed increased coupling with fusiform cortex during failed encoding on search trials, and this was generally the case for both left and right PPC. As mentioned in the introduction, the PPC is known to contribute TDA resources to bias processing of incoming sensory inputs (Desimone \& Duncan, 1995; Kastner \& Ungerleider, 2001; Sengpiel \& Hübener, 1999; Ungerleider, 2000), and BOLD signals in primary visual cortex appear to be positively associated with visual search demand (Chen et al., 2008; Maximo, Neupane, Saxena, Joseph, \& Kana, 2016; Na, Bi, Tjan, Liu, \& Fang, 2018). However, there is also evidence that the PPC contributes a switch signal to shift attention towards different regions of space in an object-sensitive (rather than purely spatial) manner (Shomstein \& Behrmann, 2006). Given the role of the fusiform cortex in object processing, it is likely that this pattern of functional connectivity reflects dPPC-mediated top-down control of attention towards objects at different spatial locations - as would be expected during visual search. Importantly, encoding failure on search trials might therefore have been partly due to difficulty in distinguishing the target from distractors based on its semantic category, requiring distributed visual attention across multiple objects in space to resolve this between-object conflict, thereby resulting in less attention directed to the target. We therefore propose that the DAN upregulates activity in modality-specific regions to foster task execution during higher task demand, especially in the service of between-object conflict, and incidentally at the expense of encoding.

In further support of the view that this reflects increases in topdown demand, this analysis also showed increased connectivity with preSMA (though this did not survive correction for multiple PPI analyses) which, as mentioned above, is a region of the frontoparietal control network involved with response conflict and monitoring (Cieslik et al., 2015; Mostofsky \& Simmonds, 2008), suggesting a complex interaction between perceptual, attentional, and control regions which would be expected during increased demand (Shashidhara, Mitchell, Erez, \& Duncan, 2019).

In contrast to previous suggestions, this shows that processes are not necessarily associated with successful encoding, nor are processes necessarily associated with failed encoding; the picture is likely more nuanced. For example, the suggested negative effects of BUA processing on encoding (Uncapher et al., 2011) might have been nullified in the current experiment by the demands of the semantic categorisation task. On the other hand, while processes might generally benefit encoding, increasing demand for target identification and or target-distractor discrimination appears to call for increases in top-down control that prioritise task execution at the expense of information encoding. These are testable hypotheses that future work should explore.

As a post hoc exploration of the observed subsequent memory effects, seeds in bilateral LOC, FG, and right hippocampus were each selected to test whether they showed changes in functional connectivity that depended on the attention manipulation. Of these five seeds, both left and right LOC showed an effect, suggesting that attention might only influence the connectivity of regions early in the object processing hierarchy, as bilateral FG and right hippocampus are involved in increasingly complex representations.

The LOC seed displayed increased coupling with FG during successful encoding of search compared with capture trials, and this effect was observed bilaterally: left LOC coupled with right, while right LOC coupled with bilateral FG. Both the LOC and FG fall within part of the ventral visual stream (Grill-Spector, Kourtzi, \& Kanwisher, 2001; Kravitz et al., 2013), and meta-analytic data from the Neurosynth database (www.neurosynth.org; Wager, 2011) showed that BOLD signal correlation with fusiform cortex is a reliable finding for both the left $(r=0.38)$ and right $(r=0.44)$ LOC peaks observed in the current study. As mentioned above, the LOC is known to be involved with the processing of perceptual shape (Kim, Biederman, Lescroart, \&
Hayworth, 2009; Kourtzi \& Kanwisher, 2001; Malikovic et al., 2016; Orlov \& Zohary, 2018), while the FG processes object semantic categories and features (Burgund \& Marsolek, 1997; Grill-Spector \& Weiner, 2014; Marsolek, 1999; Marsolek \& Burgund, 2008; McMenamin et al., 2015; Rosenke et al., 2018).

Evidence for both a representational gradient within the ventral visual network, as well as direct LOC-FG functional connectivity, was previously demonstrated in a study investigating the processing of low (coarse/simple) vs high (fine/complex) spatial frequency stimuli (Rotshtein, Vuilleumier, Winston, Driver, \& Dolan, 2007). It was found that bilateral LOC was more sensitive to manipulations among low rather than high spatial frequency images, while the opposite was true in other regions (inferior occipital \& temporal gyri); however, the right FG was sensitive to both. Crucially, connectivity analyses revealed a direct influence of LOC (coarse stimulus representation) on the responses of the FG (both coarse and well-defined stimuli). They concluded that the different regions within the ventral visual network process stimuli at different spatial scales and, importantly for the current study, outputs from the LOC project to the FG where both low and high spatial frequency information converge to produce a more integrated representation of the stimulus. The increase in LOC-FG connectivity in the current study may therefore index the more detailed elaboration of stimuli based on unique stimulus features, by more effectively integrating coarse stimulus shape (LOC) with higher level semantic and feature associations (FG). Ultimately, the more integrated a stimulus becomes during encoding, the sharper and more pattern-separated the representation can become (Pidgeon \& Morcom, 2016), leading to a more durable memory. That this effect was seen only during TDA trials suggests that increased top-down demand (mediated by the DAN) upregulates this LOC-FG coupling in order to resolve stimulus competition, which was largely absent on capture trials.

In short, LOC-FG coupling during encoding may be an index of the differentiation of individual representations as they are integrated across lower perceptual and higher semantic levels within the ventral stream. As post hoc explorations are inherently biased, further research is needed to test this directly, for example by varying the perceptual ambiguity of objects with the hypothesis that LOC-FG connectivity increases with object discriminability (e.g. Flounders, González-García, Hardstone, \& He, 2019; Summerfield et al., 2006).

\section{Summary \& conclusions}

The present study made use of a novel experimental task to test how top-down and bottom-up attention differentially contribute to memory encoding. First, we replicated previous work showing a distinction between a dorsal frontoparietal system supporting top-down attention, and a ventral frontoparietal system supporting bottom-up attention. We also demonstrate the close associations between the dorsal attention and frontoparietal control networks during top-down attention, and the ventral attention and cingulo-opercular (salience) networks during bottom-up attention. Furthermore, we showed that areas of the ventral visual stream (specifically the lateral occipital cortex and fusiform gyri) were predictive of later recognition memory success. Importantly, we did not observe modulation of subsequent memory by attention in either the behavioural or the whole-brain GLM analyses. We suggest that the elaborative processing associated with the semantic categorisation task could have overshadowed any influence of attention on subsequent memory, implying that such an influence (should it exist) is likely to be small.

With that said, task-based functional connectivity analyses revealed that dorsal posterior parietal cortex - a key node of the dorsal attention network - diffusely coupled with ventral visual and fusiform cortex during unsuccessful memory encoding, reflecting distributed top-down control in the service of task execution and at the expense of encoding. Conversely, we found that the lateral occipital cortex, during successful encoding, statistically coupled with fusiform cortex during top-down 
attention search, enabling the integration of low-level perceptual and high-level semantic information that is beneficial to encoding.

Taken together, these results suggest that the dorsal attention network shows distinct patterns of functional connectivity when comparing successful and unsuccessful encoding, while the functional connectivity of ventral visual regions appears to be modulated by attention mode during encoding. These data provide further indication that functional connectivity analyses may be more sensitive to the impact of attention on encoding than univariate whole-brain responses. Finally, the known interactions between the two attention networks, along with the cognitive control networks, should also be accounted for in future work, as they display a large degree of overlap. The use of causal methods will be particularly valuable in addressing these issues specifically, and in further exploring the interplay between attention and episodic memory.

\section{CRediT authorship contribution statement}

Lewis Dunne: Conceptualization, Investigation, Methodology, Software, Formal analysis, Data curation, Visualization, Writing - original draft, Writing - review \& editing. Bertram Opitz: . : Conceptualization, Methodology, Writing - review \& editing.

\section{Declaration of Competing Interest}

All authors declare no conflicts of interest.

\section{References}

Aly, M., \& Turk-Browne, N. B. (2016). Attention promotes episodic encoding by stabilizing hippocampal representations. Proceedings of the National Academy of Sciences of the United States of America, 113(4), E420-E429. https://doi.org/10.1073/pnas. 1518931113.

Aly, M., \& Turk-Browne, N. B. (2017). How Hippocampal Memory Shapes, and Is Shaped by, Attention. The Hippocampus from Cells to Systems (pp. 369-403). Cham: Springer International Publishing. https://doi.org/10.1007/978-3-319-50406-3_12.

Awh, E., \& Jonides, J. (2001). Overlapping mechanisms of attention and spatial working memory. Trends in Cognitive Sciences, 5(3), 119-126. https://doi.org/10.1016/S13646613(00)01593-X.

Bireta, T. J., \& Mazzei, C. M. (2016). Does the isolation effect require attention? Memory and Cognition, 44(1), 1-14. https://doi.org/10.3758/s13421-015-0538-y.

Bracci, S., Ritchie, J. B., \& de Beeck, H. O. (2017). On the partnership between neural representations of object categories and visual features in the ventral visual pathway. Neuropsychologia, 105, 153-164. https://doi.org/10.1016/j.neuropsychologia.2017. 06.010 .

Bressler, S. L., \& Menon, V. (2010). Large-scale brain networks in cognition: Emerging methods and principles. Trends in Cognitive Sciences, 14(6), 277-290. https://doi.org/ 10.1016/j.tics.2010.04.004.

Bressler, S. L., Tang, W., Sylvester, C. M., Shulman, G. L., \& Corbetta, M. (2008). Topdown control of human visual cortex by frontal and parietal cortex in anticipatory visual spatial attention. $J$ Neurosci. https://doi.org/10.1523/JNEUROSCI.1776-08. 2008.

Bridge, D. J., Cohen, N. J., \& Voss, J. L. (2017). Distinct Hippocampal versus Frontoparietal Network Contributions to Retrieval and Memory-guided Exploration. Journal of Cognitive Neuroscience, 29(8), 1324-1338. https://doi.org/10.1162/jocn_a_ 01143.

Broadbent, D. E. (1958). Perception and Communication. London: Pergamon Press.

Brodeur, M. B., Dionne-Dostie, E., Montreuil, T., \& Lepage, M. (2010). The Bank of Standardized Stimuli (BOSS), a new set of 480 normative photos of objects to be used as visual stimuli in cognitive research. PloS One, 5(5), Article e10773. https://doi. org/10.1371/journal.pone.0010773.

Brodeur, M. B., Guérard, K., \& Bouras, M. (2014). Bank of Standardized Stimuli (BOSS) phase II: 930 new normative photos. PloS One, 9(9), Article e106953. https://doi. org/10.1371/journal.pone.0106953.

Buckner, R. L., \& DiNicola, L. M. (2019). The Brain's Default Network : Updated Anatomy, Physiology, and Evolving Insights. Nature Reviews Neuroscience, 1-16. https://doi. org/10.1038/s41583-019-0212-7.

Burgund, E. D., \& Marsolek, C. J. (1997). Letter-Case-Specific Priming in the Right Cerebral Hemisphere with a Form-Specific Perceptual Identification Task. Brain and Cognition, 35(2), 239-258. https://doi.org/10.1006/brcg.1997.0940.

Chen, Y., Martinez-Conde, S., Macknik, S. L., Bereshpolova, Y., Swadlow, H. A., \& Alonso, J.-M. (2008). Task difficulty modulates the activity of specific neuronal populations in primary visual cortex. Nature Neuroscience, 11(8), 974-982. https://doi.org/10. 1038/nn.2147.

Chun, M. M., \& Turk-Browne, N. B. (2007). Interactions between attention and memory. Current Opinion in Neurobiology, 17(2), 177-184. https://doi.org/10.1016/j.conb.
2007.03.005.

Cieslik, E. C., Mueller, V. I., Eickhoff, C. R., Langner, R., \& Eickhoff, S. B. (2015). Three key regions for supervisory attentional control: Evidence from neuroimaging metaanalyses. Neuroscience \& Biobehavioral Reviews, 48, 22-34. https://doi.org/10.1016/j. neubiorev.2014.11.003.

Cloutman, L. L. (2013). Interaction between dorsal and ventral processing streams: Where, when and how? Brain and Language, 127(2), 251-263. https://doi.org/10. 1016/j.bandl.2012.08.003.

Connor, C. E., \& Knierim, J. J. (2017). Integration of objects and space in perception and memory. Nature Neuroscience, 20(11), 1493-1503. https://doi.org/10.1038/nn.4657.

Corbetta, M., Kincade, J. M., Ollinger, J. M., McAvoy, M. P., \& Shulman, G. L. (2000). Voluntary orienting is dissociated from target detection in human posterior parietal cortex. Nature Neuroscience, 3(3), 292-297. https://doi.org/10.1038/73009.

Corbetta, M., Kincade, J. M., \& Shulman, G. L. (2002). Neural Systems for Visual Orienting and Their Relationships to Spatial Working Memory. Journal of Cognitive Neuroscience, 14(3), 508-523. https://doi.org/10.1162/089892902317362029.

Corbetta, M., Patel, G., \& Shulman, G. L. (2008). The Reorienting System of the Human Brain: From Environment to Theory of Mind. Neuron, 58(3), 306-324. https://doi. org/10.1016/j.neuron.2008.04.017.

Corbetta, M., \& Shulman, G. L. (2002). Control of goal-directed and stimulus-driven attention in the brain. Nature Reviews. Neuroscience, 3(3), 201-215. https://doi.org/10. $1038 /$ nrn755.

Craik, F. I. M. (2002). Levels of processing: Past, present ... and future? Memory, 10(5-6), 305-318. https://doi.org/10.1080/09658210244000135.

Craik, F. I. M., \& Lockhart, R. S. (1972). Levels of processing: A framework for memory research. Journal of Verbal Learning and Verbal Behavior, 11(6), 671-684. https://doi org /10.1016/S0022-5371(72)80001-X.

Davachi, L., Mitchell, J. P., \& Wagner, A. D. (2003). Multiple routes to memory: distinct medial temporal lobe processes build item and source memories. Proceedings of the National Academy of Sciences of the United States of America, 100(4), 2157-2162. https://doi.org/10.1073/pnas.0337195100.

Desimone, R., \& Duncan, J. (1995). Neural mechanisms of selective visual attention. Annual Review of Neuroscience, 18(1), 193-222. https://doi.org/10.1146/annurev.ne. 18.030195.001205.

Diana, R. A., Yonelinas, A. P., \& Ranganath, C. (2007). Imaging recollection and familiarity in the medial temporal lobe: A three-component model. Trends in Cognitive Sciences, 11(9), 379-386. https://doi.org/10.1016/j.tics.2007.08.001.

Dosenbach, N. U. F., Fair, D. A., Cohen, A. L., Schlaggar, B. L., \& Petersen, S. E. (2008). A dual-networks architecture of top-down control. Trends in Cognitive Sciences, 12(3), 99-105. https://doi.org/10.1016/j.tics.2008.01.001.

Dosenbach, N. U. F., Fair, D. A., Miezin, F. M., Cohen, A. L., Wenger, K. K., Dosenbach, R. A. T., ... Petersen, S. E. (2007). Distinct brain networks for adaptive and stable task control in humans. Proceedings of the National Academy of Sciences, 104(26), 11073-11078. https://doi.org/10.1073/pnas.0704320104.

Dosenbach, N. U. F., Visscher, K. M., Palmer, E. D., Miezin, F. M., Wenger, K. K., Kang, H. C., ... Petersen, S. E. (2006). A Core System for the Implementation of Task Sets. Neuron, 50(5), 799-812. https://doi.org/10.1016/j.neuron.2006.04.031.

Evrard, H. C. (2018). Von Economo and fork neurons in the monkey insula, implications for evolution of cognition. Current Opinion in Behavioral Sciences, 21, 182-190. https://doi.org/10.1016/j.cobeha.2018.05.006.

Fine, M. S., \& Minnery, B. S. (2009). Visual salience affects performance in a working memory task. The Journal of Neuroscience : The Official Journal of the Society for Neuroscience, 29(25), 8016-8021. https://doi.org/10.1523/JNEUROSCI.5503-08. 2009.

Flounders, M. W., González-García, C., Hardstone, R., \& He, B. J. (2019). Neural dynamics of visual ambiguity resolution by perceptual prior. ELife, 8. https://doi.org/10.7554/ eLife.41861.

Forster, S., \& Lavie, N. (2011). Entirely irrelevant distractors can capture and captivate attention. Psychonomic Bulletin \& Review, 18(6), 1064-1070. https://doi.org/10. 3758/s13423-011-0172-z.

Fox, M. D., Corbetta, M., Snyder, A. Z., Vincent, J. L., \& Raichle, M. E. (2006). Spontaneous neuronal activity distinguishes human dorsal and ventral attention systems. Proceedings of the National Academy of Sciences of the United States of America, 103(26), 10046-10051. https://doi.org/10.1073/pnas.0604187103.

Friston, K. J., Ashburner, J. T., Kiebel, S. J., Nichols, T. E., \& Penny, W. D. (2007). Statistical Parametric Mapping: The Analysis of Functional Brain Images. Statistical Parametric Mapping The Analysis of Functional Brain Images (Vol. 8). Elsevier/ Academic Press. Retrieved from http://books.elsevier.com/neuro/ $?$ isbn $=9780123725608 \&$ srccode $=89660$.

Gaspelin, N., \& Luck, S. J. (2018). The Role of Inhibition in Avoiding Distraction by Salient Stimuli. Trends in Cognitive Sciences, 22(1), 79-92. https://doi.org/10.1016/j tics.2017.11.001.

Gazzaley, A., \& Nobre, A. C. (2012). Top-down modulation: Bridging selective attention and working memory. Trends in Cognitive Sciences, 16(2), 129-135. https://doi.org/ 10.1016/J.TICS.2011.11.014.

Goddard, E. (2017). A step toward understanding the human ventral visual pathway. Journal of Neurophysiology, 117(3), 872-875. https://doi.org/10.1152/jn.00358. 2016.

Gordon, E. M., Laumann, T. O., Adeyemo, B., Huckins, J. F., Kelley, W. M., \& Petersen, S E. (2016). Generation and Evaluation of a Cortical Area Parcellation from RestingState Correlations. Cerebral Cortex, 26(1), 288-303. https://doi.org/10.1093/cercor/ bhu239.

Goulden, N., Khusnulina, A., Davis, N. J., Bracewell, R. M., Bokde, A. L., McNulty, J. P., \& Mullins, P. G. (2014). The salience network is responsible for switching between the default mode network and the central executive network: Replication from DCM. NeuroImage, 99, 180-190. https://doi.org/10.1016/j.neuroimage.2014.05.052. 
Grill-Spector, K., Kourtzi, Z., \& Kanwisher, N. (2001). The lateral occipital complex and its role in object recognition. Vision Research, 41(10-11), 1409-1422. https://doi. org/10.1016/S0042-6989(01)00073-6.

Grill-Spector, K., \& Weiner, K. S. (2014). The functional architecture of the ventral temporal cortex and its role in categorization. Nature Reviews. Neuroscience, 15(8), 536-548. https://doi.org/10.1038/nrn3747.

Halamish, V. (2018). Can very small font size enhance memory? Memory \& Cognition, 46(6), 979-993. https://doi.org/10.3758/s13421-018-0816-6.

Ham, T., Leff, A., de Boissezon, X., Joffe, A., \& Sharp, D. J. (2013). Cognitive control and the salience network: An investigation of error processing and effective connectivity. The Journal of Neuroscience : The Official Journal of the Society for Neuroscience, 33(16), 7091-7098. https://doi.org/10.1523/JNEUROSCI.4692-12.2013.

Han, S. W., Eaton, H. P., \& Marois, R. (2019). Functional Fractionation of the Cinguloopercular Network: Alerting Insula and Updating Cingulate. Cerebral Cortex, 29(6), 2624-2638. https://doi.org/10.1093/cercor/bhy130.

Han, S. W., \& Marois, R. (2014). Functional fractionation of the stimulus-driven attention network. Journal of Neuroscience, 34(20), 6958-6969. https://doi.org/10.1523/ JNEUROSCI.4975-13.2014.

Holroyd, C. B., Nieuwenhuis, S., Yeung, N., Nystrom, L., Mars, R. B., Coles, M. G. H., \& Cohen, J. D. (2004). Dorsal anterior cingulate cortex shows fMRI response to internal and external error signals. Nature Neuroscience, 7(5), 497-498. https://doi.org/10. 1038/nn1238.

Holroyd, C. B., \& Umemoto, A. (2016). The research domain criteria framework: The case for anterior cingulate cortex. Neuroscience \& Biobehavioral Reviews, 71, 418-443. https://doi.org/10.1016/J.NEUBIOREV.2016.09.021.

Hopfinger, J. B., Buonocore, M. H., \& Mangun, G. R. (2000). The neural mechanisms of top-down attentional control. Nature Neuroscience, 3(3), 284-291. https://doi.org/ $10.1038 / 72999$.

Humphrey, K., \& Underwood, G. (2009). Domain knowledge moderates the influence of visual saliency in scene recognition. British Journal of Psychology (London, England : 1953), 100(Pt 2), 377-398. https://doi.org/10.1348/000712608X344780.

Igelström, Kajsa M., \& Graziano, Michael S. A. (2017). The inferior parietal lobule and temporoparietal junction: A network perspective. Neuropsychologia, 105, 70-83. https://doi.org/10.1016/j.neuropsychologia.2017.01.001.

Kastner, S., \& Ungerleider, L. G. (2001). The neural basis of biased competition in human visual cortex. Neuropsychologia, 39(12), 1263-1276. https://doi.org/10.1016/S00283932(01)00116-6.

Kerzel, D., \& Schönhammer, J. (2013). Salient stimuli capture attention and action. Attention, Perception, \& Psychophysics, 75(8), 1633-1643. https://doi.org/10.3758/ s13414-013-0512-3.

Kim, H. (2011). Neural activity that predicts subsequent memory and forgetting: A metaanalysis of 74 fMRI studies. NeuroImage, 54(3), 2446-2461. https://doi.org/10.1016/ j.neuroimage.2010.09.045.

Kim, H. (2014). Involvement of the dorsal and ventral attention networks in oddball stimulus processing: A meta-analysis. Human Brain Mapping, 35(5), 2265-2284. https://doi.org/10.1002/hbm.22326.

Kim, H. (2017). Brain regions that show repetition suppression and enhancement: A metaanalysis of 137 neuroimaging experiments. Human Brain Mapping, 38(4), 1894-1913. https://doi.org/10.1002/hbm.23492.

Kim, J. G., Biederman, I., Lescroart, M. D., \& Hayworth, K. J. (2009). Adaptation to objects in the lateral occipital complex (LOC): Shape or semantics? Vision Research, 49(18), 2297-2305. https://doi.org/10.1016/J.VISRES.2009.06.020.

Kirwan, C. B., Wixted, J. T., \& Squire, L. R. (2008). Activity in the medial temporal lobe predicts memory strength, whereas activity in the prefrontal cortex predicts recollection. The Journal of Neuroscience : The Official Journal of the Society for Neuroscience, 28(42), 10541-10548. https://doi.org/10.1523/JNEUROSCI.3456-08. 2008.

Kourtzi, Z., \& Kanwisher, N. (2001). Representation of Perceived Object Shape by the Human Lateral Occipital Complex. Science, 293(5534), 1506-1509. https://doi.org/ 10.1126/science.1061133.

Koutstaal, W., Buckner, R. L., Wagner, A. D., Rotte, M., Maril, A., \& Schacter, D. L. (2001). Perceptual specificity in visual object priming: Functional magnetic resonance imaging evidence for a laterality difference in fusiform cortex. Retrieved from Neuropsychologia, 39(2), 184-199. http://eutils.ncbi.nlm.nih.gov/entrez/eutils/ elink.fcgi? dbfrom $=$ pubmed\&id $=11163375 \&$ retmode $=$ ref\& $\mathrm{cmd}=$ prlinks \%5Cnpapers3://publication/uuid/DA94A953-2FAA-4E65-A250-0A4852349C98.

Kravitz, D. J., Saleem, K. S., Baker, C. I., Ungerleider, L. G., \& Mishkin, M. (2013). The ventral visual pathway: An expanded neural framework for the processing of object quality. Trends in Cognitive Sciences, 17(1), 26. https://doi.org/10.1016/J.TICS.2012. 10.011.

Kucyi, A., Hodaie, M., \& Davis, K. D. (2012). Lateralization in intrinsic functional connectivity of the temporoparietal junction with salience- and attention-related brain networks. Journal of Neurophysiology, 108(12), 3382-3392. https://doi.org/10.1152/ jn.00674.2012.

Kurth, F., Zilles, K., Fox, P. T., Laird, A. R., \& Eickhoff, S. B. (2010). A link between the systems: Functional differentiation and integration within the human insula revealed by meta-analysis. Brain Structure \& Function, 214(5-6), 519-534. https://doi.org/10. 1007/s00429-010-0255-z.

Malikovic, A., Amunts, K., Schleicher, A., Mohlberg, H., Kujovic, M., Palomero-Gallagher, N., ... Zilles, K. (2016). Cytoarchitecture of the human lateral occipital cortex: Mapping of two extrastriate areas hOc4la and hOc4lp. Brain Structure and Function, 221(4), 1877-1897. https://doi.org/10.1007/s00429-015-1009-8.

Marcus, D. S., Harms, M. P., Snyder, A. Z., Jenkinson, M., Wilson, J. A., Glasser, M. F., ... Van Essen, D. C. (2013). Human Connectome Project informatics: Quality control, database services, and data visualization. NeuroImage, 80, 202-219. https://doi.org/ 10.1016/j.neuroimage.2013.05.077.
Marek, S., \& Dosenbach, N. U. F. (2018). The frontoparietal network: Function, electrophysiology, and importance of individual precision mapping. Retrieved from www.dialogues-cns.org. Dialogues Clin Neurosci, 20.

Marsolek, C. J. (1999). Dissociable neural subsystems underlie abstract and specific object recognition. Psychological Science, 10(2), 111-118. https://doi.org/10.1111/14679280.00117.

Marsolek, C. J., \& Burgund, E. D. (2008). Dissociable neural subsystems underlie visual working memory for abstract categories and specific exemplars. Retrieved from Cognitive, Affective \& Behavioral Neuroscience, 8(1), 17-24. http://www.ncbi.nlm.nih. gov/pubmed/18405042.

Maximo, J. O., Neupane, A., Saxena, N., Joseph, R. M., \& Kana, R. K. (2016). TaskDependent Changes in Frontal-Parietal Activation and Connectivity during Visual Search. Brain Connectivity, 6(4), 335-344. https://doi.org/10.1089/brain.2015.0343.

McMenamin, B. W., Deason, R. G., Steele, V. R., Koutstaal, W., \& Marsolek, C. J. (2015). Separability of abstract-category and specific-exemplar visual object subsystems: Evidence from fMRI pattern analysis. Brain and Cognition, 93, 54-63. https://doi.org/ 10.1016/j.bandc.2014.11.007.

Melcher, D., \& Piazza, M. (2011). The Role of Attentional Priority and Saliency in Determining Capacity Limits in Enumeration and Visual Working Memory. PLoS ONE, 6(12), Article e29296. https://doi.org/10.1371/journal.pone.0029296.

Menon, V., \& Uddin, L. Q. (2010). Saliency, switching, attention and control: A network model of insula function. Brain Structure \& Function, 214(5-6), 655-667. https://doi. org/10.1007/s00429-010-0262-0.

Milner, A. D. (2017). How do the two visual streams interact with each other? Experimental Brain Research, 235(5), 1297-1308. https://doi.org/10.1007/s00221 017-4917-4.

Mostofsky, S. H., \& Simmonds, D. J. (2008). Response Inhibition and Response Selection: Two Sides of the Same Coin. Journal of Cognitive Neuroscience, 20(5), 751-761. https://doi.org/10.1162/jocn.2008.20500.

Na, R., Bi, T., Tjan, B. S., Liu, Z., \& Fang, F. (2018). Effect of task difficulty on bloodoxygen-level-dependent signal: A functional magnetic resonance imaging study in a motion discrimination task. PLoS ONE, 13(6), 1-19. https://doi.org/10.1371/ journal.pone.0199440.

Naghavi, H. R., \& Nyberg, L. (2005). Common fronto-parietal activity in attention, memory, and consciousness: Shared demands on integration? Consciousness and Cognition, 14(2), 390-425. https://doi.org/10.1016/J.CONCOG.2004.10.003.

Naveh-Benjamin, Moshe, Guez, Jonathan, Hara, Yoko, Brubaker, Matthew S., \& Lowenschuss-Erlich, Iris (2014). The Effects of Divided Attention on Encoding Processes under Incidental and Intentional Learning Instructions: Underlying Mechanisms? Quarterly Journal of Experimental Psychology, 67(9), 1682-1696. https://doi.org/10.1080/17470218.2013.867517.

Nelson, S. M., Dosenbach, N. U. F., Cohen, A. L., Wheeler, M. E., Schlaggar, B. L., \& Petersen, S. E. (2010). Role of the anterior insula in task-level control and focal attention. Brain Structure \& Function, 214(5-6), 669-680. https://doi.org/10.1007/ s00429-010-0260-2.

Opitz, B. (2010). Neural binding mechanisms in learning and memory. Neuroscience and Biobehavioral Reviews, 34(7), 1036-1046. https://doi.org/10.1016/j.neubiorev.2009. 11.001.

Orlov, T., \& Zohary, E. (2018). Object Representations in Human Visual Cortex Formed Through Temporal Integration of Dynamic Partial Shape Views. The Journal of Neuroscience, 38(3), 659-678. https://doi.org/10.1523/JNEUROSCI.1318-17.2017.

Paller, K. A., \& Wagner, A. D. (2002). Observing the transformation of experience into memory. Trends in Cognitive Sciences, 6(2), 93-102. https://doi.org/10.1016/S13646613(00)01845-3.

Pidgeon, L. M., \& Morcom, A. M. (2016). Cortical pattern separation and item-specific memory encoding. Neuropsychologia, 85, 256-271. https://doi.org/10.1016/j. neuropsychologia.2016.03.026.

Posner, M. I. (2014). Orienting of attention: Then and now. Quarterly Journal of Experimental Psychology, 2006, 1-12. https://doi.org/10.1080/17470218.2014. 937446.

Power, J. D., Cohen, A. L., Nelson, S. M., Wig, G. S., Barnes, K. A., Church, J. A., ... Petersen, S. E. (2011). Functional Network Organization of the Human Brain. Neuron, 72(4), 665-678. https://doi.org/10.1016/j.neuron.2011.09.006.

Prince, S. E., Daselaar, S. M., \& Cabeza, R. (2005). Neural Correlates of Relational Memory: Successful Encoding and Retrieval of Semantic and Perceptual Associations. Journal of Neuroscience, 25(5), 1203-1210. https://doi.org/10.1523/JNEUROSCI. 2540-04.2005.

Ridderinkhof, K. R., Ullsperger, M., Crone, E. A., \& Nieuwenhuis, S. (2004). The Role of the Medial Frontal Cortex in Cognitive Control. Science, 306(5695), 443-447. https:// doi.org/10.1126/science.1100301.

Rorden, C., \& Brett, M. (2000). Stereotaxic display of brain lesions. Retrieved from Behavioural Neurology, 12(4), 191-200. http://www.ncbi.nlm.nih.gov/pubmed/ 11568431.

Rosenke, M., Weiner, K. S., Barnett, M. A., Zilles, K., Amunts, K., Goebel, R., \& GrillSpector, K. (2018). A cross-validated cytoarchitectonic atlas of the human ventral visual stream. NeuroImage, 170, 257-270. https://doi.org/10.1016/j.neuroimage. 2017.02.040.

Rotshtein, P., Vuilleumier, P., Winston, J., Driver, J., \& Dolan, R. (2007). Distinct and convergent visual processing of high and low spatial frequency information in faces. Cerebral Cortex, 17(11), 2713-2724. https://doi.org/10.1093/cercor/bhl180.

Santangelo, V., \& Macaluso, E. (2013a). The contribution of working memory to divided attention. Human Brain Mapping, 34(1), 158-175. https://doi.org/10.1002/hbm. 21430.

Santangelo, V., \& Macaluso, E. (2013b). Visual salience improves spatial working memory via enhanced parieto-temporal functional connectivity. The Journal of Neuroscience : The Official Journal of the Society for Neuroscience, 33(9), 4110-4117. https://doi.org/ 
10.1523/JNEUROSCI.4138-12.2013.

Schmidt, S. R., \& Schmidt, C. R. (2017). Revisiting von Restorff's early isolation effect. Memory \& Cognition, 45(2), 194-207. https://doi.org/10.3758/s13421-016-0651-6.

Schneider, W., \& Shiffrin, R. M. (1977). Controlled and automatic human information processing: I. Detection, search, and attention. Psychological Review, 84(1), 1-66. https://doi.org/10.1037/0033-295X.84.1.1.

Schreij, D., Theeuwes, J., \& Olivers, C. N. L. (2010). Abrupt onsets capture attention independent of top-down control settings II: Additivity is no evidence for filtering. Attention, Perception, and Psychophysics, 72(3), 672-682. https://doi.org/10.3758/ APP.72.3.672.

Seeley, W. W., Menon, V., Schatzberg, A. F., Keller, J., Glover, G. H., Kenna, H., ... Greicius, M. D. (2007). Dissociable Intrinsic Connectivity Networks for Salience Processing and Executive Control. Journal of Neuroscience, 27(9), 2349-2356. https:// doi.org/10.1523/jneurosci.5587-06.2007.

Sengpiel, F., \& Hübener, M. (1999). Visual perception: Spotlight on the primary visual cortex. Current Biology, 9(9), R318-R321. https://doi.org/10.1016/S0960-9822(99) 80202-4.

Shashidhara, S., Mitchell, D. J., Erez, Y., \& Duncan, J. (2019). Progressive Recruitment of the Frontoparietal Multiple-demand System with Increased Task Complexity, Time Pressure, and Reward. Journal of Cognitive Neuroscience, 31(11), 1617-1630. https:// doi.org/10.1162/jocn_a_01440.

Shepard, R. N. (1967). Recognition memory for words, sentences, and pictures. Journal of Verbal Learning and Verbal Behavior, 6(1), 156-163. https://doi.org/10.1016/S00225371(67)80067-7.

Shomstein, S., \& Behrmann, M. (2006). Cortical systems mediating visual attention to both objects and spatial locations. Proceedings of the National Academy of Sciences of the United States of America, 103(30), 11387-11392. https://doi.org/10.1073/pnas. 0601813103.

Simons, J. S., Koutstaal, W., Prince, S., Wagner, A. D., \& Schacter, D. L. (2003). Neural mechanisms of visual object priming: Evidence for perceptual and semantic distinctions in fusiform cortex. NeuroImage, 19(3), 613-626. https://doi.org/10.1016/ S1053-8119(03)00096-X.

Sokolov, E. N. (1963). Perception and the conditioned reflex. New York, NY: Pergamon.

Squire, L. R., Stark, C. E. L., \& Clark, R. E. (2004). the Medial Temporal Lobe. Annual Review of Neuroscience, 27(1), 279-306. https://doi.org/10.1146/annurev.neuro.27. 070203.144130 .

Standing, L. (1973). Learning 10,000 pictures. Quarterly Journal of Experimental Psychology, 25(2), 207-222. https://doi.org/10.1080/14640747308400340.

Strange, B. A., Henson, R. N. A., Friston, K. J., \& Dolan, R. J. (2000). Brain mechanisms for detecting perceptual, semantic, and emotional deviance. NeuroImage, 12(4), 425-433. https://doi.org/10.1006/nimg.2000.0637.

Summerfield, C., Egner, T., Greene, M., Koechlin, E., Mangels, J., \& Hirsch, J. (2006). Predictive codes for forthcoming perception in the frontal cortex. Science, 314(5803), 1311-1314. https://doi.org/10.1126/science.1132028.

Summerfield, J. J., Lepsien, J., Gitelman, D. R., Mesulam, M. M., \& Nobre, A. C. (2006). Orienting Attention Based on Long-Term Memory Experience. Neuron, 49(6), 905-916. https://doi.org/10.1016/j.neuron.2006.01.021.

Sunny, M. M., \& von Mühlenen, A. (2013). Attention capture by abrupt onsets: Re-visiting the priority tag model. Frontiers in Psychology, 4, 958. https://doi.org/10.3389/fpsyg. 2013.00958.

Theeuwes, J. (1991). Exogenous and endogenous control of attention: The effect of visual onsets and offsets. Perception \& Psychophysics, 49(1), 83-90. https://doi.org/10. 3758/BF03211619.

Theeuwes, J. (1992). Perceptual selectivity for color and form. Perception \& Psychophysics, 51(6), 599-606. https://doi.org/10.3758/BF03211656.

Thompson, K. G., Biscoe, K. L., \& Sato, T. R. (2005). Neuronal basis of covert spatial attention in the frontal eye field. The Journal of Neuroscience : The Official Journal of the Society for Neuroscience, 25(41), 9479-9487. https://doi.org/10.1523/ JNEUROSCI.0741-05.2005.

Treisman, A., \& Gelade, G. (1980). A feature-integration theory of attention. Cognitive Psychology, 12(1), 97-136. https://doi.org/10.1016/0010-0285(80)90005-5.

Treisman, A., \& Gormican, S. (1988). Feature analysis in early vision: Evidence from search asymmetries. Retrieved from Psychological Review, 95(1), 15-48. http://www. ncbi.nlm.nih.gov/pubmed/3353475.

Uddin, L. Q. (2015). Salience processing and insular cortical function and dysfunction. Nature Reviews Neuroscience, 16(1), 55-61. https://doi.org/10.1038/nrn3857.

Uddin, L. Q., Nomi, J. S., Hébert-Seropian, B., Ghaziri, J., \& Boucher, O. (2017). Structure and Function of the Human Insula. Journal of Clinical Neurophysiology, 34(4), 300-306. https://doi.org/10.1097/WNP.0000000000000377.

Uncapher, M. R., Hutchinson, J. B., \& Wagner, A. D. (2011). Dissociable Effects of TopDown and Bottom-Up Attention during Episodic Encoding. Journal of Neuroscience, 31(35), 12613-12628. https://doi.org/10.1523/JNEUROSCI.0152-11.2011.

Uncapher, M. R., \& Rugg, M. D. (2005). Effects of divided attention on fMRI correlates of memory encoding. Journal of Cognitive Neuroscience, 17(12), 1923-1935. https://doi org/10.1162/089892905775008616.

Uncapher, M. R., \& Rugg, M. D. (2009). Selecting for Memory? The Influence of Selective Attention on the Mnemonic Binding of Contextual Information. Journal of Neuroscience, 29(25), 8270-8279. https://doi.org/10.1523/jneurosci.1043-09.2009.

Uncapher, M. R., \& Wagner, A. D. (2009). Posterior parietal cortex and episodic encoding: Insights from fMRI subsequent memory effects and dual-attention theory. Neurobiology of Learning and Memory, 91(2), 139-154. https://doi.org/10.1016/j. nlm.2008.10.011.

Ungerleider, L. G. (2000). Mechanisms of Visual Attention in the Human Cortex. Annual Review of Neuroscience, 23(1), 315-341. https://doi.org/10.1146/annurev.neuro.23. 1.315 .

Vossel, S., Geng, J. J., \& Fink, G. R. (2014). Dorsal and ventral attention systems: Distinct neural circuits but collaborative roles. The Neuroscientist : A Review Journal Bringing Neurobiology, Neurology and Psychiatry, 20(2), 150-159. https://doi.org/10.1177/ 1073858413494269.

Wager, T. D. (2011). NeuroSynth: A new platform for large-scale automated synthesis of human functional neuroimaging data. Frontiers in Neuroinformatics, 5. https://doi. org/10.3389/conf.fninf.2011.08.00058.

West Chanon, V., \& Hopfinger, J. B. (2008). Memory's grip on attention: The influence of item memory on the allocation of attention. Visual Cognition, 16(2-3), 325-340. https://doi.org/10.1080/13506280701459026.

Wolfe, J. M., \& Horowitz, T. S. (2017). Five factors that guide attention in visual search. Nature Human Behaviour, 1(3), 1-8. https://doi.org/10.1038/s41562-017-0058.

Yantis, S., \& Hillstrom, A. P. (1994). Stimulus-driven attentional capture: Evidence from equiluminant visual objects. Retrieved from Journal of Experimental Psychology. Human Perception and Performance, 20(1), 95-107.

Yantis, Steven (1993). Stimulus-Driven Attentional Capture. Current Directions in Psychological Science, 2(5), 156-161. https://doi.org/10.1111/1467-8721. ep10768973.

Yantis, Steven, \& Jonides, J. (1984). Abrupt visual onsets and selective attention: Evidence from visual search. Journal of Experimental Psychology: Human Perception and Performance, 10(5), 601-621. https://doi.org/10.1037/0096-1523.10.5.601.

Yeo, B. T. T., Krienen, F. M., Sepulcre, J., Sabuncu, M. R., Lashkari, D., Hollinshead, M., .. Buckner, R. L. (2011). The organization of the human cerebral cortex estimated by intrinsic functional connectivity. Journal of Neurophysiology, 106(3), 1125-1165. https://doi.org/10.1152/jn.00338.2011.

Zhou, Y., Friston, K. J., Zeidman, P., Chen, J., Li, S., \& Razi, A. (2018). The Hierarchical Organization of the Default, Dorsal Attention and Salience Networks in Adolescents and Young Adults. Cerebral Cortex, 28(2), 726-737. https://doi.org/10.1093/cercor/ bhx307. 